\title{
Monotonicity and Li-Yau-Hamilton Inequalities
}

\author{
Lei $\mathrm{Ni}$
}

\begin{abstract}
This is a survey article for Surveys in Differential Geometry series on the subject of Li-Yau-Hamilton type differential inequalities and related monotonicity formulae.
\end{abstract}

\section{Introduction}

The purpose of this essay is to give an expository account on various sharp estimates of Li-Yau-Hamilton type for solutions to geometric evolution equations and their relation to various important monotonicity formulae in the subject.

The Li-Yau-Hamilton type estimates in the geometric evolution equations are originally called differential Harnack estimates since they imply the celebrated Harnack estimates for the parabolic equations originated with the work of Moser $[\mathbf{M o}]$. In the seminar paper $[\mathbf{L Y}]$ P. Li and S.-T. Yau first proved a gradient estimate for positive solutions to the heat equation via the maximum principle and derived a sharp form of Harnack estimate by integrating the proven gradient estimate on space-time paths. Even though its proof has its root in the corresponding earlier works on geometric elliptic equations (cf. [ChY1, ChY2, Y], etc.), this gradient estimate differs from its elliptic analogue fundamentally by its sharpness as well as its broader impact to the study of nonlinear geometric evolution equations. Later on, the similar technique was employed by R. Hamilton in the study of Ricci flow $[\mathbf{H 2}, \mathbf{H 3}]$, as well as the hypersurface mean curvature flow $[\mathbf{H} 4]$. Based on this history and its great impact towards the study of geometric evolution equations, in $[\mathbf{N T}]$, the class of sharp differential inequalities which yield a Harnack type estimate by the path-integration, started to be called LiYau-Hamilton type estimates. Soon after Hamilton's work the corresponding results for the Kähler-Ricci flow and Gauss curvature flow (Yamabe flow) were proved by H.-D. Cao $[\mathbf{C o}]$ and B. Chow $[\mathbf{C h 1}, \mathbf{C h 2}]$ respectively. Later USA.

The author was supported in part by NSF Grants and an Alfred P. Sloan Fellowship, 
in $[\mathbf{A n 1} 1$, the result was established for a general class of hypersurface flows by B. Andrews.

On the other hand, Hamilton also generalized Li-Yau's original estimate for the linear heat equation to a matrix form on a class of Riemannian manifolds with nonnegative sectional curvature [H5]. More importantly he derived several important monotonicity formulae out of his matrix estimate $[\mathbf{H 6}]$.

In the last decade there were a few further developments along this direction, [CC1, CC2, CH, NT, CN, N3, N4]. Many applications in geometry have also been discovered. The most spectacular one is the LiYau-Hamilton type estimate for the conjugate heat equation (coupled with the Ricci flow) and its related entropy monotonicity formula discovered by G. Perelman $[\mathbf{P}]$ (see also [N5]). Perelman's inequality and its relation with the reduced volume monotonicity suggest a profound connection between the monotonicity formulae and Li-Yau-Hamilton type estimates. It is one of our purposes here to convey this connection.

Due to its expository nature, in this paper we shall only include a complete proof of a statement in the cases either the stated result appears in the first time, or we are compelled by the simplicity of the original arguments. In the rest of the cases, we shall just give a outline or the key steps.

Here is how we organize this paper. In Section 2 we discuss various LiYau-Hamilton inequalities on geometric evolution equations. In Section 3 we discuss how various monotonicity formulae can be derived out of the Li-Yau-Hamilton inequalities. In Section 4 we discuss how monotonicity formulae in turn suggest new Li-Yau-Hamilton type inequalities. The order of the appearance of the results may not necessarily follow the chronological order in which the results were proved. Instead we present them in the order which we feel is the most natural and logical. Due to the lack of expertise and the limited time allowed for writing this article we have to omit the applications of Li-Yau-Hamilton inequality altogether, as well as other various important topics, which we shall list in the section of comments for interested parties. Through out the paper $n$ denotes the dimension of a Riemannian manifold $M^{n}$ and $m$ denotes the complex dimension of a Kähler manifold $M^{m}(n=2 m)$.

\section{Li-Yau-Hamilton type inequalities}

The most miraculous result is Hamilton's matrix Li-Yau-Hamilton (which we shall abbreviate as LYH later) for Ricci flow of metrics with a bounded nonnegative curvature operator. However its proof as well as the formulation is motivated by the corresponding simpler consideration on linear heat equations. We also found that it is more suggestive to start with the simpler case since its understanding often sheds lights on the more technically complicated nonlinear setting. Hence we start with the solutions to the linear heat equation. 
2.1. Linear heat equation. Through out this subsection, let $(M, g)$ be a fixed Riemannian manifold. The main concern is on the positive solution to

$$
\left(\frac{\partial}{\partial t}-\Delta\right) u(x, t)=0
$$

A. Li-Yau's gradient estimate for the linear heat equation. In $[\mathbf{L Y}]$, the following result was proved.

Theorem 2.1. Let $(M, g)$ be a complete Riemannian manifold. Assume that on the ball $B(o, 2 R)$, $\operatorname{Ric}(M) \geq-k$. Then for any $\alpha>1$, we have that

$$
\begin{aligned}
\sup _{B(o, R)}\left(\frac{|\nabla u|^{2}}{u^{2}}-\alpha \frac{u_{t}}{u}\right) \leq & \frac{C \alpha^{2}}{R^{2}}\left(\frac{\alpha^{2}}{\alpha^{2}-1}+\sqrt{k} R\right) \\
& +\frac{n \alpha^{2} k}{2(\alpha-1)}+\frac{n \alpha^{2}}{2 t} .
\end{aligned}
$$

If $(M, g)$ has nonnegative Ricci, letting $R \rightarrow \infty,(2.2)$ gives the clean estimate (a Hamilton-Jacobi inequality):

$$
\frac{|\nabla u|^{2}}{u^{2}}-\frac{u_{t}}{u} \leq \frac{n}{2 t}
$$

This estimate is sharp in the sense that the equality satisfied for some $\left(x_{0}, t_{0}\right)$ implies that $(M, g)$ is isometric to $\mathbb{R}^{n}[\mathbf{N 5}]$. It can also be easily checked that if $u$ is the fundamental solution on $\mathbb{R}^{n}$ given by the formula $\frac{1}{(4 \pi t)^{\frac{n}{2}}} \exp \left(-\frac{|x|^{2}}{4 t}\right)$, then the equality holds in (2.3). It was observed by Li-Yau that by integration over the path jointing $\left(x_{1}, t_{1}\right)$ to $\left(x_{2}, t_{2}\right)$ with $t_{2}>t_{1},(2.3)$ gives

$$
u\left(x_{1}, t_{1}\right) \leq\left(\frac{t_{2}}{t_{1}}\right)^{\frac{n}{2}} u\left(x_{2}, t_{2}\right) \exp \left(\frac{r^{2}\left(x_{1}, x_{2}\right)}{4\left(t_{2}-t_{1}\right)}\right)
$$

which is a sharp form of Harnack estimates for parabolic equations.

Despite the fundamental importance of Theorem 2.1, its proof is elementary via the maximum principle for parabolic equations by computing $\left(\frac{\partial}{\partial t}-\Delta\right) F$ with $F=\frac{|\nabla u|^{2}}{u^{2}}-\alpha \frac{u}{u_{t}}$. The localized estimate (2.2) is possible due to a term of $-F^{2}$ appearing in the resulting estimation of $\left(\frac{\partial}{\partial t}-\Delta\right) F$. The Ricci curvature comes into play due to the commutation of the differentiations as in the regular Bochner formula $[\mathbf{S Y}]$. In the special case that $(M, g)$ has nonnegative Ricci curvature, the proof of (2.3) is based on the following computation. Let $Q:=u\left(\Delta \log u+\frac{n}{2 t}\right)$. Then

$$
\left(\frac{\partial}{\partial t}-\Delta\right) Q=\frac{2}{u}\left|\Upsilon_{i j}\right|^{2}-\frac{2}{t} Q+\frac{2}{u} R_{i j} \nabla_{i} u \nabla_{j} u
$$


where

$$
\Upsilon_{i j}=\nabla_{i} \nabla_{j} u+\frac{u}{2 t} g_{i j}-\frac{u_{i} u_{j}}{u}=u\left(-\nabla_{i} \nabla_{j} f+\frac{1}{2 t} g_{i j}\right) .
$$

with $f$ defined by $u=\frac{e^{-f}}{(4 \pi t)^{n / 2}} \cdot\left(Q=g^{i j} \Upsilon_{i j}.\right)$

B. The differential estimates on the fundamental solution. In [N2], motivated by the work of $[\mathbf{P}]$ the following result was proved.

Theorem 2.2. Let $(M, g)$ be a complete Riemannian manifold with nonnegative Ricci curvature. Let $u(x, t)=H(x, t ; y, o)$ be the positive heat kernel. Then

$$
t\left(2 \Delta f-|\nabla f|^{2}\right)+f-n \leq 0
$$

where $u=\frac{e^{-f}}{(4 \pi t)^{\frac{n}{2}}}$.

The estimate (2.5) is a Li-Yau-Hamilton type since combining with the heat equation $2|\nabla f|^{2}-2 \Delta f+2 f_{t}+\frac{n}{t}=0$ we have a Hamilton-Jacobi inequality

$$
|\nabla f|^{2}+2 f_{t}+\frac{f}{t} \leq 0
$$

For $\left(x_{2}, t_{2}\right)$ and $\left(x_{1}, t_{1}\right)$ with $t_{2}>t_{1}$ we have that

$$
\begin{aligned}
\sqrt{t_{2}} f\left(x_{2}, t_{2}\right)-\sqrt{t_{1}} f\left(x_{1}, t_{1}\right) & =\int_{t_{1}}^{t_{2}} \frac{d}{d t}(\sqrt{t} f(\gamma(t), t)) d t \\
& =\int_{t_{1}}^{t_{2}} \sqrt{t}\left(f_{t}+\frac{f}{2 t}+\left\langle\nabla f, \gamma^{\prime}(t)\right\rangle\right) d t \\
& \leq \frac{1}{2} \int_{t_{1}}^{t_{2}} \sqrt{t}\left|\gamma^{\prime}(t)\right|^{2} d t
\end{aligned}
$$

for any path joining from $x_{1}$ to $x_{2}$. This gives a Harnack type estimate:

(2.7) $\sqrt{t_{2}} f\left(x_{2}, t_{2}\right)-\sqrt{t_{1}} f\left(x_{1}, t_{1}\right) \leq \inf _{\gamma} \frac{1}{2} \int_{t_{1}}^{t_{2}} \sqrt{t}\left|\gamma^{\prime}(t)\right|^{2} d t=\frac{r^{2}\left(x_{1}, x_{2}\right)}{4\left(\sqrt{t_{2}}-\sqrt{t_{1}}\right)}$.

Since $\lim _{t \rightarrow 0} \sqrt{t} f(o, t) \leq 0$ from that $\lim _{t \rightarrow 0} u(x, t)=\lim _{t \rightarrow 0} \frac{e^{-\frac{\sqrt{t} f(x, t)}{\sqrt{t}}}}{(4 \pi t)^{\frac{n}{2}}}=$ $\delta_{o}(x)$, we have that

$$
f\left(x_{2}, t_{2}\right) \leq \frac{r^{2}\left(o, x_{2}\right)}{4 t_{2}}
$$

This is equivalent to the heat kernel comparison theorem of Cheeger and Yau [CY]. 
The curious reader may ask why the estimate (2.5) is not proved for arbitrary positive solutions. The simple answer is that it is no longer true in such generality. This can be seen for those solutions which are smooth on $M \times[0, T)$. The proof of the result requires both the point-wise computation as Theorem 2.1 (precisely (2.4)) as well as an entropy formula together with some integral estimates. Hence we postpone a more detailed account to a later section. However, there is a geometric consideration which indicates that (2.5) is a natural one and is related to (2.3). It was known that

$$
\lim _{t \rightarrow 0} 4 t f=r^{2}(o, x)
$$

if $u$ is the heat kernel. Rewriting (2.3) as

$$
t(2 \Delta f)-n \leq 0
$$

one therefore deduces the Laplacian comparison theorem:

$$
\Delta r^{2} \leq 2 n
$$

Define $\hat{L}(x, t):=4 t f(x, t)$. Then $(2.3)$ amounts to $\Delta \hat{L} \leq 2 n$. Therefore one can view it as a generalized/space-time version Laplacian comparison theorem. Writing (2.5) in terms of $\hat{L}$ we have that

$$
\Delta \hat{L}+\hat{L}_{t} \leq 2 n \text {. }
$$

In this sense, (2.5) is a space-time Laplacian comparison theorem. The sharpness of (2.5) follows from that the equality holds for some $(x, t)$ with $t>0$ if and only if $M$ is isometric to $\mathbb{R}^{n}[\mathbf{N 5}]$.

C. The matrix LYH inequalities. We found that keeping in mind the connection between the LYH type estimate and the comparison theorem on distance functions is beneficial. The following result of Hamilton [H5] corresponds to the Hessian comparison theorem.

Theorem 2.3. Assume that $(M, g)$ is a complete Riemannian manifold with nonnegative sectional curvature and parallel Ricci curvature. Then

$$
\nabla_{i} \nabla_{j} \log u+\frac{1}{2 t} g_{i j} \geq 0
$$

Noting the trace of (2.10) is just (2.3). The extra assumption that $(M, g)$ has parallel Ricci is quite restrictive, which essentially means that $(M, g)$ is Einstein. When $(M, g)$ is a Kähler manifold this assumption can be dropped and the nonnegativity of the sectional curvature can be relaxed to the nonnegativity of the bisectional curvature. This was observed in $[\mathbf{C N}]$.

THEOREM 2.4. Let $(M, g)$ be a complete Kähler manifold with nonnegative holomorphic bisectional curvature. Then

$$
\nabla_{i} \nabla_{\bar{j}} \log u+\frac{1}{t} g_{i \bar{j}} \geq 0 .
$$


Both (2.10) and (2.11) yield the Hessian comparison theorem for the distance function as illustrated before. In the next section we shall show how they can suggest estimates for the Ricci/Kähler-Ricci flow.

The proof is as usual via the maximum principle. This time one needs the tensor maximum principle of Hamilton developed in $[\mathbf{H 1}]$. The rest is on computing

$$
\left(\frac{\partial}{\partial t}-\Delta\right)\left(\nabla_{i} \nabla_{j} \log u+\frac{1}{2 t} g_{i j}\right)
$$

and grouping the resulting terms.

D. LYH inequality on $(1,1)$ forms. The above results are all on the positive solution to the heat equation. The curious reader may ask if there is any such result for solutions to a parabolic system. There is one indeed [N3]. But we have to restrict ourselves to the complex Kähler manifolds.

Let $h(x, t)=\sqrt{-1} h_{\alpha \bar{\beta}} d z^{\alpha} \wedge d z^{\bar{\beta}}$ be a real $(1,1)$ form satisfying the Lichnerowicz-Laplacian heat equation:

$$
\left(\frac{\partial}{\partial t}-\Delta\right) h_{\gamma \bar{\delta}}=R_{\beta \bar{\alpha} \gamma \bar{\delta}} h_{\alpha \bar{\beta}}-\frac{1}{2}\left(R_{\gamma \bar{s}} h_{s \bar{\delta}}+R_{s \bar{\delta}} h_{\gamma \bar{s}}\right) .
$$

We assume that $h_{\alpha \bar{\beta}}(x, t)$ is semi-positive definite (denoted briefly as $\left.h_{\alpha \bar{\beta}}(x, t) \geq 0\right)$ and that $M$ has nonnegative bisectional curvature. For any $(1,0)$ vector field $V$ we define

$$
\begin{aligned}
Z_{h}(x, t)= & \frac{1}{2}\left(g^{\alpha \bar{\beta}} \nabla_{\bar{\beta}} \operatorname{div}(h)_{\alpha}+g^{\gamma \bar{\delta}} \nabla_{\gamma} \operatorname{div}(h)_{\bar{\delta}}\right)+g^{\alpha \bar{\beta}} \operatorname{div}(h)_{\alpha} V_{\bar{\beta}} \\
& +g^{\gamma \bar{\delta}} \operatorname{div}(h)_{\bar{\delta}} V_{\gamma}+g^{\alpha \bar{\beta}} g^{\gamma \bar{\delta}} h_{\alpha \bar{\delta}} V_{\bar{\beta}} V_{\gamma}+\frac{H}{t}
\end{aligned}
$$

Here

$$
\operatorname{div}(h)_{\alpha}=g^{\gamma \bar{\delta}} \nabla_{\gamma} h_{\alpha \bar{\delta}}, \quad \operatorname{div}(h)_{\bar{\delta}}=g^{\alpha \bar{\beta}} \nabla_{\bar{\beta}} h_{\alpha \bar{\delta}}
$$

and

$$
H=g^{\alpha \bar{\beta}} h_{\alpha \bar{\beta}} .
$$

In the context where the meaning is clear we drop the subscript $h$ in $Z_{h}$.

THEOREM 2.5. Let $M$ be a complete Kähler manifold with nonnegative holomorphic bisectional curvature. Let $h_{\alpha \bar{\beta}}(x, t) \geq 0$ be a symmetric $(1,1)$ tensor satisfying (1.1) on $M \times(0, T)$. Assume that for any $\epsilon^{\prime}>0$,

$$
\int_{\epsilon^{\prime}}^{T} \int_{M} e^{-a r^{2}(x)}\|h\|^{2} d v d t<\infty .
$$


Then

$$
Z(x, t) \geq 0
$$

for any $(1,0)$ vector $V$. If $Z\left(x_{0}, t_{0}\right)=0$ for some point $\left(x_{0}, t_{0}\right)$ with $t_{0}>0$ and $h_{\alpha \bar{\beta}}(x, t)>0$, then $M$ is flat.

The proof is via the maximum principle as usual. Instead of explaining on that we choose to formulate the result in more conventional way for the people who are more familiar with the complex geometric notations. Recall the Hodge-Kodaira Laplacian

$$
\Delta^{\prime \prime}=\bar{\partial} \bar{\partial}^{*}+\bar{\partial}^{*} \bar{\partial}
$$

The standard Kodaira-Bochner formula gives

$$
\begin{gathered}
-\Delta^{\prime \prime} h=\Delta_{L} h \doteqdot \Delta h_{\alpha \bar{\beta}}+R_{\beta \bar{\alpha} \gamma \bar{\delta}} h_{\alpha \bar{\beta}}-\frac{1}{2}\left(R_{\gamma \bar{s}} h_{s \bar{\delta}}+R_{s \bar{\delta}} h_{\gamma \bar{s}}\right), \\
\partial^{*} h=-\operatorname{div}(h)_{\bar{\delta}} d z^{\bar{\delta}}, \quad \bar{\partial}^{*} h=\operatorname{div}(h)_{\alpha} d z^{\alpha}
\end{gathered}
$$

and

$$
g^{\alpha \bar{\beta}} \nabla_{\bar{\beta}} \operatorname{div}(h)_{\alpha}=-\partial^{*} \bar{\partial}^{*} h, \quad g^{\alpha \bar{\beta}} \nabla_{\alpha} \operatorname{div}(h)_{\bar{\beta}}=\bar{\partial}^{*} \partial^{*} h .
$$

Hence the result concludes that for $h \geq 0$ satisfying the heat equation

$$
\left(\frac{\partial}{\partial t}+\Delta^{\prime \prime}\right) h=0
$$

the scalar quantity

$$
Z_{h}=\frac{1}{2}\left(-\partial^{*} \bar{\partial}^{*}+\bar{\partial}^{*} \partial^{*}\right) h+\bar{\partial}^{*} h(V)-\partial^{*} h(\bar{V})+h(V, \bar{V})+\frac{\Lambda h}{t} \geq 0
$$

for any vector field $V$. Here $\Lambda h$ is the standard contraction of $h$ by the Kähler form.

When $h$ is the trace of the curvature form of a Hermitian vector bundle satisfying the Hermitian-Einstein flow, Theorem 2.5 gives a Li-Yau-Hamilton estimate for Hermitian-Einstein flow on nonnegative curved manifolds. Please see [N3] for details on this.

2.2. Ricci/Kähler-Ricci flow. For most discussion in this subsection, $(M, g(t))$ is a solution to Ricci/Kähler Ricci flow. The most important result for Ricci flow is Hamilton's [H3] matrix LYH inequality.

A. Matrix LYH inequalities on curvature. The following fundamental result for Ricci flow was proved by Hamilton [H3] (see also [H2]).

TheOREM 2.6. If $\left(M^{n}, g(t)\right), t \in[0, T)$, is a solution to the Ricci flow with nonnegative curvature operator, namely $R_{i j k l} U_{i j} U_{k l} \geq 0$ for all 2-forms, 
and if $\left(M^{n}, g(t)\right)$ is either compact or complete noncompact with bounded curvature, then for any 1 -form $W \in C^{\infty}\left(\Lambda^{1} M\right)$ and 2 -form $U \in C^{\infty}\left(\Lambda^{2} M\right)$ we have

$$
Z(U, W) \doteqdot M_{i j} W_{i} W_{j}+2 P_{p i j} U_{p i} W_{j}+R_{i p j q} U_{i p} U_{j q} \geq 0
$$

Here

$$
P_{k i j} \doteqdot \nabla_{k} R_{i j}-\nabla_{i} R_{k j}
$$

and the symmetric 2-tensor $M$ is defined by

$$
M_{i j} \doteqdot \Delta R_{i j}-\frac{1}{2} \nabla_{i} \nabla_{j} R+2 R_{i k j l} R_{k l}-R_{i p} R_{p j}+\frac{1}{2 t} R_{i j}
$$

The original paper $[\mathbf{H 3}]$ is still the best place to read the proof of this result. The expression is suggested by playing with gradient expanding soliton equations. This again is well explained in $[\mathbf{H 3}]$. As in Li-Yau's case, the expression of the estimate is obtained by the computation of the special solutions (grouping various gradient terms of the heat kernel on the Euclidean space $\mathbb{R}^{n}$ yields the LYH expression for the linear heat equation, grouping the soliton equation and its various covariant differentiations on an gradient expanding soliton yields the matrix LYH expression for the Ricci flow). The LYH expression usually vanishes identically on the special solutions. However it is highly nontrivial, if not a miracle, to come up as complicated an expression as (2.18) and conclude that it has a sign for the general solutions/spaces.

The Kähler analogue of the above result was proved by Cao $[\mathbf{C o}]$. Define $(2.21)$

$$
\tilde{Y}(X)_{\alpha \bar{\beta}} \doteqdot \frac{\partial}{\partial t} R_{\alpha \bar{\beta}}+R_{\alpha \bar{\gamma}} R_{\gamma \bar{\beta}}+\nabla_{\gamma} R_{\alpha \bar{\beta}} X^{\gamma}+\nabla_{\bar{\gamma}} R_{\alpha \bar{\beta}} X^{\bar{\gamma}}+R_{\alpha \bar{\beta} \gamma \bar{\delta}} X^{\gamma} X^{\bar{\delta}}+\frac{R_{\alpha \bar{\beta}}}{t}
$$

for any $(1,0)$-vector $X=X^{\gamma} \frac{\partial}{\partial z^{\gamma}}$ and where $X^{\bar{\gamma}} \doteqdot \overline{X^{\gamma}}$.

THEOREM 2.7. If $\left(\mathcal{M}^{n}, g(t)\right)$ is a complete solution to the Kähler-Ricci flow with bounded nonnegative bisectional curvature, then

$$
\left(\tilde{Y}(X)_{\alpha \bar{\beta}}\right) \geq 0
$$

for any $(1,0)$-vector $X$.

B. The linear trace LYH inequalities. Recall that the Lichnerowicz Laplacian acting on symmetric 2-tensors is given by

$$
\Delta_{L} h_{i j}=\Delta h_{i j}+2 R_{i k j l} h_{k l}-R_{i k} h_{j k}-R_{j k} h_{i k} .
$$

The following result was proved by Chow and Hamilton $[\mathbf{C H}]$. 
TheOREM 2.8. Suppose $\left(M^{n}, g(t)\right), t \in[0, T)$, is a complete solution of Ricci flow with bounded nonnegative curvature operator. Assume that $h(x, t)$ with $h(0) \geq 0$, is a solution to

$$
\frac{\partial}{\partial t} h=\Delta_{L} h
$$

and $|h(t)|$ is bounded. Then $h(t) \geq 0$ for $t \in[0, T)$ and for any vector $X$ we have

$$
Z(X) \doteqdot \nabla_{i} \nabla_{j} h_{i j}+R_{i j} h_{i j}+2\left(\nabla_{j} h_{i j}\right) X_{i}+h_{i j} X_{i} X_{j}+\frac{H}{2 t} \geq 0,
$$

where $H \doteqdot g^{i j} h_{i j}$.

If $h_{i j}$ is the Ricci tensor, one can check that (2.23) holds. Hence the above theorem implies the trace form of (2.18) (choosing $U=X \wedge W$ and tracing the variable $W$ ). Also (2.23) is satisfied by the variational tensors of a family of solutions to Ricci flow in a certain sense. Namely, (2.23) is a linearization of the Ricci flow. This explains the term linear trace.

In $[\mathbf{N T}]$, with the motivation of study the Liouville property of plurisubharmonic functions, the Kähler analogue of the above was proved. Let $\left(\mathcal{M}^{n}, g(t)\right), t \in[0, T)$, be a complete noncompact solution of the Kähler-Ricci flow with bounded nonnegative bisectional curvature. Let $h=$ $\sqrt{-1} h_{\alpha \bar{\beta}} d z^{\alpha} \wedge d z^{\bar{\beta}}$ be real $(1,1)$ form satisfying $(2.16)$. Define

$$
\begin{aligned}
Z(h, V) \doteqdot & \frac{1}{2} g^{\alpha \bar{\beta}}\left(\nabla_{\bar{\beta}} \operatorname{div}(h)_{\alpha}+\nabla_{\alpha} \operatorname{div}(h)_{\bar{\beta}}\right)+R_{\alpha \bar{\beta}} h_{\beta \bar{\alpha}} \\
& +g^{\alpha \bar{\beta}}\left(\operatorname{div}(h)_{\alpha} V_{\bar{\beta}}+\operatorname{div}(h)_{\bar{\beta}} V_{\alpha}\right)+h_{\alpha \bar{\beta}} V_{\beta} V_{\bar{\alpha}}+\frac{H}{t},
\end{aligned}
$$

where $V$ is a vector field of type $(1,0), H$ and $\operatorname{div}(h)_{\alpha}$ are as before.

THEOREM 2.9. Suppose that $\left(\mathcal{M}^{n}, g(t)\right), t \in[0, T)$, is a complete solution of the Kähler-Ricci flow with bounded nonnegative bisectional curvature and $h \geq 0$ satisfying (2.16) and (2.14). Then

$$
Z(h, V) \geq 0
$$

on $\mathcal{M} \times[0, T)$ for any vector field $V$ of type $(1,0)$.

As before, expressed in terms operators $\bar{\partial}^{*}$ and $\partial^{*}$, the result asserts (2.26)

$$
Z=\frac{1}{2}\left(-\partial^{*} \bar{\partial}^{*}+\bar{\partial}^{*} \partial^{*}\right) h+\bar{\partial}^{*} h(V)-\partial^{*} h(\bar{V})+\operatorname{Ric}(h)+h(V, \bar{V})+\frac{\Lambda h}{t} \geq 0 .
$$

Here $\operatorname{Ric}(h)$ is the contraction of $h$ by the Ricci form. 
C. A matrix LYH inequality for forward conjugate heat equation. Now we consider the forward conjugate heat equation:

$$
\left(\frac{\partial}{\partial t}-\Delta\right) u(x, t)=R(x, t) u(x, t) .
$$

Here $R(x, t)$ is the scalar curvature. The following result was proved in $[\mathbf{N 4}]$.

THEOREM 2.10. Let $(M, g(t))$ be a solution to Kähler-Ricci flow defined on $M \times[0, T]$ (for some $T>0$ ) with nonnegative bisectional curvature. In the case that $M$ is complete noncompact, assume further that the bisectional curvature is bounded on $M \times[0, T]$. Let $u$ be a positive solution to (2.27). Then

$$
u_{\alpha \bar{\beta}}+\frac{u}{t} g_{\alpha \bar{\beta}}+u R_{\alpha \bar{\beta}}+u_{\alpha} V_{\bar{\beta}}+u_{\bar{\beta}} V_{\alpha}+u V_{\alpha} V_{\bar{\beta}} \geq 0
$$

for any $(1,0)$ vector field $V$.

By choosing the minimizing vector $V$ in (2.28) we have

$$
\nabla_{\alpha} \nabla_{\bar{\beta}} \log u+R_{\alpha \bar{\beta}}+\frac{1}{t} g_{\alpha \bar{\beta}} \geq 0 .
$$

When $m=1$, namely $M$ is a Riemann sphere, we have the following estimate which is very similar to Li-Yau's (2.3)

$$
\Delta \log u+R+\frac{1}{t} \geq 0
$$

This result was first proved by Chow and Hamilton $[\mathbf{C H}]$.

The result was discovered by the interpolation consideration which shall be explained next.

D. Interpolations. The following estimates was proved by Chow [Ch3], which links the Li-Yau estimate to the linear trace estimate (2.30) when $M$ is the Riemann sphere with positive curvature.

Proposition 2.11. Given $\varepsilon>0$, if $\left(M^{2}, g(t)\right)$ is a solution to the $\varepsilon$-Ricci flow

$$
\frac{\partial}{\partial t} g_{i j}=-2 \varepsilon R_{i j}=-\varepsilon R g_{i j}
$$

on a closed surface with $R>0$ and if $u$ is a positive solution to

$$
\frac{\partial}{\partial t} u=\Delta u+\varepsilon R u,
$$

then

$$
\frac{\partial}{\partial t} \log u-|\nabla \log u|^{2}+\frac{1}{t}=\Delta \log u+\varepsilon R+\frac{1}{t} \geq 0
$$


There are two different high dimensional generalizations of the above result. They are all in the category of Kähler manifolds. The first one proved in [N4] is straightforward and connects Theorem 2.10 with Theorem 2.4 (more precisely, connects (2.11) with (2.29).

For any $\epsilon>0$, we consider the $\epsilon$-Kähler-Ricci flow:

$$
\frac{\partial}{\partial t} g_{\alpha \bar{\beta}}(x, t)=-\epsilon R_{\alpha \bar{\beta}}(x, t) .
$$

Consider the positive solution $u$ to the parabolic equation:

$$
\left(\frac{\partial}{\partial t}-\Delta\right) u(x, t)=\epsilon R(x, t) u(x, t) .
$$

We shall call (2.35) forward conjugate heat equation, since it is the adjoint of the backward heat equation $\left(\frac{\partial}{\partial t}+\Delta\right) v=0$.

THEOREM 2.12. Assume that the complete solution $(M, g(t))$ (defined on $M \times[0, T]$ for some $T>0$ ) to (2.34) has nonnegative bisectional curvature. In the case that $M$ is noncompact, assume further that the bisectional curvature of $g(t)$ is uniformly bounded on $M \times[0, T]$. Let $u$ be a positive solution to (2.35). Then

$$
\nabla_{\alpha} \nabla_{\bar{\beta}} \log u+\epsilon R_{\alpha \bar{\beta}}+\frac{u}{t} g_{\alpha \bar{\beta}} \geq 0 .
$$

It is obvious that Theorem 2.12 generalizes (2.33). The second one proved in $[\mathbf{N 3}]$, connects Theorem 2.5 with Theorem 2.9 (more precisely (2.17) and $(2.26))$.

THEOREM 2.13. Let $(M, g(t))$ be a solution to (2.34) with bounded nonnegative bisectional curvature. Let $h(x, t) \geq 0$ be a real $(1,1)$ form satisfying (2.16). Then

$Z_{h, \epsilon}=\frac{1}{2}\left(-\partial^{*} \bar{\partial}^{*}+\bar{\partial}^{*} \partial^{*}\right) h+\bar{\partial}^{*} h(V)-\partial^{*} h(\bar{V})+\epsilon \operatorname{Ric}(h)+h(V, \bar{V})+\frac{\Lambda h}{t} \geq 0$.

Moreover, the equality holds for some $t>0$ implies that $(M, g(t))$ is an expanding gradient soliton, provided that $h_{\alpha \bar{\beta}}(x, t)>0$ and $M$ is simplyconnected.

It is less obvious that Theorem 2.13 also generalizes (2.33). To see this, first observe that $H=\Lambda h$ satisfies

$$
\left(\frac{\partial}{\partial t}-\Delta\right) H=\epsilon \operatorname{Ric}(h)
$$

which generalizes (2.32). Now we restrict ourselves to the case in which $h$ is closed. Using the Kähler identities

$$
\partial \Lambda-\Lambda \partial=\bar{\partial}^{*}, \quad \bar{\partial} \Lambda-\Lambda \bar{\partial}=-\partial^{*}
$$


we have that

$$
Z_{h, \epsilon}=\Delta H+\partial H(V)+\bar{\partial} H(\bar{V})+\epsilon \operatorname{Ric}(h)+h(V, \bar{V})+\frac{H}{t} \geq 0 .
$$

In the case $m=1$, this gives the estimate (2.33), noticing that $-\Delta^{\prime}=$ $-\Delta^{\prime \prime}=\Delta$.

Since (2.38) and (2.40) make sense for any $(p, p)$ form $h \geq 0$, it is natural question to ask if (2.40) holds for $(p, p)$ form $h \geq 0$. Theorem 2.12 shows that it is the case for $(m, m)$ forms. We conjecture that it is the case for general nonnegative $(p, p)$ forms.

E. LYH for the conjugate heat equation. Assume that $(M, g(t))$ is a solution to Ricci flow on $M \times[0, T]$. Now we consider the conjugate heat equation:

$$
\left(\frac{\partial}{\partial \tau}-\Delta+R\right) u(x, \tau)=0
$$

Here $\tau=T-t$. This equation is the adjoint of the heat equation $\left(\frac{\partial}{\partial t}-\Delta\right) v=0$. In $[\mathbf{P}]$, the following estimate was discovered by Perelman.

THEOREM 2.14. Assume that $u=\frac{e^{-f}}{(4 \pi \tau)^{\frac{n}{2}}}$ is the fundamental solution to (2.41) with $u(o, 0)=\delta_{o}(x)$. Then

$$
\tau\left(2 \Delta f-|\nabla f|^{2}+R\right)+f-n \leq 0 .
$$

Note that the result is equivalent to the space time Lapalacian comparison

$$
\Delta \hat{L}+\hat{L}_{\tau} \leq 2 n
$$

with $\hat{L}=4 \tau f$. Comparing the above with Theorem 2.2, the most striking part is that the result holds without any curvature assumption. The detailed proof of Theorem 2.14 was given in [N5]. The key equation is the following one discovered by Perelman

$$
\left(\frac{\partial}{\partial \tau}-\Delta+R\right) V=-2 \tau\left|R_{i j}+\nabla_{i} \nabla_{j} f-\frac{1}{2 \tau} g_{i j}\right|^{2} u
$$

where

$$
V=\left[\tau\left(2 \Delta f-|\nabla f|^{2}+R\right)+f-n\right] u .
$$

The equation (2.44) can be derived from the following simpler one which resembles (2.4)

$$
\left(\frac{\partial}{\partial \tau}-\Delta+R\right) v_{0}=-2\left|R_{i j}+f_{i j}\right|^{2} u
$$

if $v_{0}=\left(2 \Delta f-|\nabla f|^{2}+R\right) u$. 
Combining (2.42) with (2.41) we have that

$$
2 f_{\tau}+|\nabla f|^{2}-R+\frac{f}{\tau} \leq 0
$$

which yields for any $\gamma(\tau)$ joining $\left(x_{1}, \tau_{1}\right)$ with $\left(x_{2}, \tau_{2}\right)$,

$$
\begin{aligned}
\sqrt{\tau_{2}} f\left(x_{2}, \tau_{2}\right)-\sqrt{\tau_{1}} f\left(x_{1}, \tau_{1}\right) & =\int_{\tau_{1}}^{\tau_{2}} \sqrt{\tau}\left(f_{\tau}+\frac{f}{2 \tau}+\left\langle\nabla f, \gamma^{\prime}\right\rangle\right) d \tau \\
& \leq \frac{1}{2} \int_{\tau_{1}}^{\tau_{2}} \sqrt{\tau}\left(\left|\gamma^{\prime}\right|^{2}+R\right) d \tau .
\end{aligned}
$$

Now observing as before that $\lim _{\tau \rightarrow 0} \sqrt{\tau} f(o, \tau) \leq 0$, we have that

$$
f(x, \bar{\tau}) \leq \frac{1}{2 \sqrt{\bar{\tau}}} \inf _{\gamma} \int_{0}^{\bar{\tau}} \sqrt{\tau}\left(\left|\gamma^{\prime}\right|^{2}+R\right) d \tau .
$$

The right hand side is called reduced distance, denoted by $\ell(x, \tau)$. There exists another way of proving (2.47) via the fact that

$$
\left(\frac{\partial}{\partial \tau}-\Delta+R\right) \hat{u}(x, \tau) \leq 0
$$

where $\hat{u}(x, \tau)=\frac{e^{-\ell(x, \tau)}}{(4 \pi \tau)^{\frac{n}{2}}}$ satisfying that $\lim _{\tau \rightarrow 0} \hat{u}(x, \tau)=\delta_{o}(x)$. For the purpose of better presentation we work with $t$ instead. Assume that $H(x, t ; y, T)$ (with $t \leq T$ ) is the fundamental solution of (2.41) and $h(y, s ; x, t)$ (with $s \geq t$ ) is the fundamental solution to the heat equation. The well-known duality asserts that

$$
h(z, s ; x, t)=H(x, t ; z, s) .
$$

Now we can check that

$$
I(s)=\int_{M} h(z, s ; x, t) \hat{u}(z, s ; y, T) d \mu_{s}(z)
$$

is monotone increasing in $s$. Here $\hat{u}(z, s ; y, T)$ corresponds to the reduced distance starting from $(y, T)$. Hence

$$
\begin{aligned}
H(x, t ; y, T) & =h(y, T ; x, t)=\lim _{s \rightarrow T} I(s) \\
& \geq \lim _{s \rightarrow t} I(s)=\hat{u}(x, t ; y, T)
\end{aligned}
$$

from which (2.47) follows easily.

2.3. Hypersurface flows in $\mathbb{R}^{n+1}$. The LYH estimates for the hypersurface flow in $\mathbb{R}^{n+1}$ were first proved for mean curvature flow by Hamilton $[\mathbf{H} 4]$ and Gauss curvature flow by Chow [Ch1]. Here we present the work of Andrews on much more general class of hypersurface flows, which is a 
lot less computational than the original works of Hamilton and Chow. Our presentation follows that of [An1, An2].

First we collect some formulae and results needed on the hypersurface's curvature flow. In our discussion we assume that $X_{0}: M \rightarrow \mathbb{R}^{n+1}$ is a immersed closed $n$-dimensional hypersurface. Consider the 1-family of smooth hypersurfaces $X: M \times[0, T) \rightarrow \mathbb{R}^{n+1}$ satisfying

$$
\frac{\partial X}{\partial t}(x, t)=-f(x, t) \nu(x, t)
$$

where $f(x, t)$ is a smooth function and $\nu(x, t)$ is the outer unit normal. We assume that $M_{t}=X(M, t)$ are compact convex hypersurfaces. The following are well-known.

Let $g_{i j}$ be the induced metric, $d \mu$ be the induced measure. Then

$$
\begin{aligned}
\frac{\partial}{\partial t} g_{i j} & =-2 f h_{i j} \\
\frac{\partial}{\partial t} d \mu & =-f H d \mu \\
\frac{\partial}{\partial t} h_{i j} & =\nabla_{i} \nabla_{j} f-f h_{i k} h_{j l} g^{k l} .
\end{aligned}
$$

Usually, we write that $f(x, t)=F(W(x, t))$, where $W=\left\{h_{j}^{i}\right\}$ is the Weingartan map. Then

$$
\begin{aligned}
\frac{\partial}{\partial t} W & =g^{*}\left(\operatorname{Hess}_{\nabla} F\right)+F W^{2} \\
\frac{\partial}{\partial t} H & =\operatorname{tr}\left(g^{*}\left(\operatorname{Hess}_{\nabla} F\right)\right)+F|A|^{2} \\
\frac{\partial}{\partial t} \nu & =\nabla F \\
\frac{\partial}{\partial t} F & =\dot{F}\left(g^{*}\left(\operatorname{Hess}_{\nabla} F\right)\right)+F \dot{F}\left(W^{2}\right) .
\end{aligned}
$$

A. The support function. The support function is very useful for the convex hypersurface flow. Especially because it can be used to reparametrize the surface via the Gauss map and greatly reduce the computation in deriving the LYH inequalities for the hypersurfaces flow.

Let $s(z): \mathbb{S}^{n} \rightarrow \mathbb{R}$ be the support function of $M$ (precisely, $X: M \rightarrow$ $\left.\mathbb{R}^{n+1}\right)$. It can be defined by

$$
s(z)=\left\langle z, X\left(\nu^{-1}(z)\right)\right\rangle
$$

where $\nu(x)=\nu(X(x))$, namely the normal of the image. We may recover the immersion after the reparametrization by

$$
\bar{X}(z)=s(z) z+\bar{\nabla} s
$$


where $\bar{\nabla}$ is the standard connection of $\mathbb{S}^{n}$. For simplicity when there is no confusion we just write $X(z)$. Geometrically it is very clear that

$$
X(z)=s(z) z+a(z)
$$

for some vector $a(z)$, tangent to the sphere (normal to $z$ ). Just differentiate we have that

$$
\begin{aligned}
d X(u) & =\langle\bar{\nabla} s, u\rangle z+s u+D_{u} a \\
& =\langle\bar{\nabla} s, u\rangle z+s u+\bar{\nabla}_{u} a-\bar{g}(u, a) z
\end{aligned}
$$

Here $\bar{g}$ is the metric of the sphere $D$ is the direction derivative of $\mathbb{R}^{n+1}$. Noticing that $d X(u)$ is tangent to the sphere we have that

$$
\langle\bar{\nabla} s, u\rangle=\bar{g}(u, a) \quad \text { hence } \quad a=\bar{\nabla} s .
$$

Namely we have the equation

$$
X(z)=s(z) z+\bar{\nabla} s .
$$

More precisely we have shown that $X(x)=\bar{X}\left(\nu^{-1}(x)\right)=s(z) z+\bar{\nabla} s$. If we extend $s$ to $\mathbb{R}^{n+1}$ homogenously as a degree 1 function we have that $D_{z} s=s$. Hence

which implies that

$$
D s=s(z) z+\bar{\nabla} s
$$

$$
X(z)=D s .
$$

This gives the immersion from the support function.

For the parametrization via Gauss map as above, it changes the computation on $M$ to computation with respect to the fixed standard metric of $\mathbb{S}^{n}$.

LEMMA 2.15.

$$
W^{-1}=\bar{g}^{*}(\bar{\nabla} \bar{\nabla} s+s \text { id }) .
$$

Proof. Observe that $\nu(X(z))=z$. Hence we have that $W^{-1}=d X$. Now using (2.56) we just compute

$$
d X(u)=\bar{\nabla}_{u} \bar{\nabla} s+s u
$$

and we have the result.

The following lemma shows the relation on the time derivatives with respect to different parametrizations.

LEMMA 2.16. Let $Q(x, t)$ and $\bar{Q}(z, t)$ be the quantities related by $Q(x, t)=$ $\bar{Q}(z(x, t), t)$. Then

$$
\frac{\partial Q}{\partial t}=\frac{\partial \bar{Q}}{\partial t}+h^{-1}(\nabla F, \nabla Q)
$$

Here $h^{-1}$ stands for the inverse of the second fundamental form. 
Proof. Using the connection $X(x, t)=\bar{X}(z(x, t), t)$ we have that

$$
\begin{aligned}
-F(x, t) \nu(x, t) & =\frac{\partial}{\partial t} X(x, t) \\
& =\frac{\partial}{\partial t} \bar{X}(z, t)+d \bar{X}\left(\frac{\partial z}{\partial t}\right) .
\end{aligned}
$$

Recalling $\bar{X}(z, t)=s(z, t) z+\bar{\nabla} s$ we have that

$$
\frac{\partial}{\partial t} \bar{X}(z, t)=\left(\frac{\partial}{\partial t} s\right) z+\bar{\nabla}\left(\frac{\partial}{\partial t} s\right) .
$$

By comparing the normal and tangential components we have that

$$
\begin{aligned}
\frac{\partial}{\partial t} s & =-F=-\Phi(z, t) \\
\frac{\partial z}{\partial t} & =-(d \bar{X})^{-1}\left(\bar{\nabla}\left(\frac{\partial}{\partial t} s\right)\right)=(d \bar{X})^{-1}(\bar{\nabla} F)=(d \bar{X})^{-1}(\bar{\nabla} \Phi) .
\end{aligned}
$$

Here when $F$ is viewed as a function on $\mathbb{S}^{n}$ we write as $\Phi(z, t)$. Then

$$
\begin{aligned}
\frac{\partial}{\partial t} s & =-\Phi \\
\frac{\partial z}{\partial t} & =(d \bar{X})^{-1}(\bar{\nabla} \Phi) .
\end{aligned}
$$

Now we have that

$$
\begin{aligned}
\frac{\partial Q}{\partial t} & =\frac{\partial \bar{Q}}{\partial t}+\left\langle\bar{\nabla} \bar{Q}, \frac{\partial z}{\partial t}\right\rangle \\
& =\frac{\partial \bar{Q}}{\partial t}+\left\langle\bar{\nabla} \bar{Q},(d \bar{X})^{-1}(\bar{\nabla} \Phi)\right\rangle .
\end{aligned}
$$

To get what we need we use the following observations. First we have also (from (2.54))

$$
\frac{\partial z}{\partial t}=d X(\nabla F)
$$

Hence

$$
\left\langle\bar{\nabla} \bar{Q}, \frac{\partial z}{\partial t}\right\rangle=\langle\bar{\nabla} \bar{Q}, d X(\nabla F)\rangle=g\left(\nabla F, d X^{-1}(\bar{\nabla} \bar{Q})\right) .
$$

On the other hand for any tangent vector $u$ we have that

$$
\begin{aligned}
g(\nabla Q, u) & =u Q=d Q(u) \\
& =d \bar{Q}(d \nu(d X(u))) \\
& =\langle d \nu(d X(u)), \bar{\nabla} Q\rangle \\
& =g\left(W(u), d X^{-1}(\bar{\nabla} \bar{Q})\right) .
\end{aligned}
$$


Let $u W^{-1}(\nabla F)$ we have that

$$
g\left(\nabla Q, W^{-1}(\nabla F)\right)=\left\langle\bar{\nabla} \bar{Q}, \frac{\partial z}{\partial t}\right\rangle .
$$

This proves the claim.

When $Q=F($ then $\bar{Q}(z, t)=\Phi(z, t)$, we have that

$$
\frac{\partial}{\partial t} F-h^{-1}(\nabla F, \nabla F)=\frac{\partial}{\partial t} \Phi .
$$

Note the left hand side often appears in the differential Harnack inequality.

It turns out that it is better that we consider $\Phi(X)=F\left(X^{-1}\right)$. In terms of the support function we examine the equation (2.60) for several flows.

Mean curvature flow: $F(W)=\sum \lambda_{i}$, where $W$ is the Weingartan map, hence $\Phi(A)=\sum \frac{1}{\mu_{i}}$, where $\mu_{i}$ are eigenvalues of $A=W^{-1}=\bar{g}^{*} \operatorname{Hess}_{\bar{\nabla}} s+s$ id. Then the mean curvature flow can be expressed as

$$
\frac{\partial s}{\partial t}=-\operatorname{tr}\left(\left(\bar{g}^{*} \operatorname{Hess}_{\bar{\nabla}} s+s \mathrm{id}\right)^{-1}\right) .
$$

Gauss curvature flow: $F(W)=\Pi \lambda_{i}$, where $W$ is the Weingartan map, hence $\Phi(A)=\Pi \frac{1}{\mu_{i}}$, where $\mu_{i}$ are eigenvalues of $A=W^{-1}=\bar{g}^{*} \operatorname{Hess}_{\bar{\nabla}} s+s$ id. Then the Gauss curvature flow can be expressed as

$$
\frac{\partial s}{\partial t}=-\frac{1}{\operatorname{det}\left(\bar{g}^{*} \operatorname{Hess}_{\bar{\nabla}} s+s \mathrm{id}\right)}
$$

which was the form more studied from PDE point of view.

Harmonic mean curvature flow: $F(W)=\frac{1}{\sum \frac{1}{\lambda_{i}}}$, where $W$ is the Weingartan map, hence $\Phi(A)=\frac{1}{\sum \mu_{i}}$, where $\mu_{i}$ are eigenvalues of $A=$ $W^{-1}=\bar{g}^{*} \operatorname{Hess}_{\bar{\nabla}} s+s$ id. Then the harmonic mean curvature flow can be expressed as

$$
\frac{\partial s}{\partial t}=-\frac{1}{\bar{\Delta} s+n s}
$$

The $\frac{1}{H}$-curvature flow: $F(W)=-\frac{1}{\sum \lambda_{i}}$, where $W$ is the Weingartan map, hence $\Phi(A)=-\frac{1}{\sum \frac{1}{\mu_{i}}}$, where $\mu_{i}$ are eigenvalues of $A=W^{-1}=\bar{g}^{*} \operatorname{Hess}_{\bar{\nabla}} s+$ $s$ id. Then the $\frac{1}{H}$-curvature flow mean curvature flow can be expressed as

$$
\frac{\partial s}{\partial t}=\frac{1}{\operatorname{tr}\left(\left(\bar{g}^{*} \operatorname{Hess}_{\bar{\nabla}} s+s \mathrm{id}\right)^{-1}\right)} .
$$

B. The LYH inequality. Using the support function and parametrization via the Gauss map, we have the following result. 
Theorem 2.17. Assume that $-\Phi$ is $\alpha$-concave for some $\alpha<0$. Then

$$
\frac{\partial \Phi}{\partial t}+\frac{\alpha \Phi}{(\alpha-1) t} \geq 0
$$

Using (2.62) we have that

$$
\frac{\partial}{\partial t} F-h^{-1}(\nabla F, \nabla F)+\frac{\alpha F}{(\alpha-1) t} \geq 0 .
$$

$\Psi: T^{*} \mathbb{S}^{n} \otimes T \mathbb{S}^{n} \rightarrow \mathbb{R}^{n}$ is called $\alpha$-concave if

$$
\ddot{\Psi} \leq \frac{\alpha-1}{\alpha \Psi} \dot{\Psi} \otimes \dot{\Psi} .
$$

We first check the applicability of the theorem to two example curvature flows: the Gauss curvature flow and the mean curvature flow.

Recall the following useful computational lemma.

Lemma 2.18. When A is diagonal,

$$
\begin{aligned}
\dot{F}(A) & =\operatorname{diag}\left(\frac{\partial f(\lambda)}{\partial \lambda_{1}}, \cdots, \frac{\partial f(\lambda)}{\partial \lambda_{n}}\right) ; \\
\ddot{F}(A)(X, X) & =\sum_{p, q} \frac{\partial^{2} f}{\partial \lambda_{p} \lambda_{q}} X_{p}^{p} X_{q}^{q}+\sum_{p \neq q} \frac{\frac{\partial f}{\partial \lambda_{p}}-\frac{\partial f}{\partial \lambda_{q}}}{\lambda_{p}-\lambda_{q}}\left(X_{p}^{q}\right)^{2} .
\end{aligned}
$$

Hence if $\frac{\partial f}{\partial \lambda_{i}}>0$ at some $A$, then $\dot{F}$ is positive definite. And if $f$ is convex (concave), $F$ is convex (concave).

Mean curvature flow: $F(W)=\sum \lambda_{i}$, where $W$ is the Weingartan map, hence $\Phi(A)=\sum \frac{1}{\mu_{i}}$, where $\mu_{i}$ are eigenvalues of $A=W^{-1}=g^{*} \operatorname{Hess}_{\bar{\nabla}} s+s$ id. $\Psi=-\Phi=-\sum \frac{1}{\mu_{i}}$. Using the computational lemma we have that

$$
\begin{aligned}
\ddot{\Psi}(X, X) & =\sum_{p} \frac{2}{\mu_{p}^{3}}\left|X_{p}^{p}\right|^{2}-\sum_{p \neq q} \frac{\mu_{p}+\mu_{q}}{\mu_{p}^{2} \mu_{q}^{2}}\left|X_{p}^{q}\right|^{2} \\
& =-\sum_{p q} \frac{\mu_{p}+\mu_{q}}{\mu_{p}^{2} \mu_{q}^{2}}\left|X_{p}^{q}\right|^{2}
\end{aligned}
$$

and

$$
\begin{aligned}
\dot{\Psi}(X) & =\frac{1}{\mu_{p}^{2}} X_{p}^{p} \\
\frac{(\dot{\Psi}(X))^{2}}{\Psi} & =-\frac{\left(\sum \frac{1}{\mu_{p}^{2}} X_{p}^{p}\right)\left(\sum \frac{1}{\mu_{q}^{2}} X_{q}^{q}\right)}{\sum \frac{1}{\mu_{i}}} .
\end{aligned}
$$


Using $\mu_{p}+\mu_{q} \geq 2 \sqrt{\mu_{p} \mu_{q}}$, we have that

$$
\begin{aligned}
\sum_{p q} \frac{\mu_{p}+\mu_{q}}{\mu_{p}^{2} \mu_{q}^{2}}\left|X_{p}^{q}\right|^{2} & \geq \sum_{p q} \frac{2}{\mu_{p}^{1+1 / 2} \mu_{q}^{1+1 / 2}}\left|X_{p}^{q}\right|^{2} \\
& \geq \sum_{p} \frac{2}{\mu_{p}^{1+1 / 2} \mu_{p}^{1+1 / 2}}\left|X_{p}^{p}\right|^{2} \\
& \geq 2 \frac{\left(\sum \frac{1}{\mu_{p}^{2}} X_{p}^{p}\right)\left(\sum \frac{1}{\mu_{q}^{2}} X_{q}^{q}\right)}{\sum \frac{1}{\mu_{i}}}
\end{aligned}
$$

Hence $\Psi$ is -1 -concave for the mean curvature flow and we have Hamilton's

$$
\frac{\partial}{\partial t} F-h^{-1}(\nabla F, \nabla F)+\frac{F}{2 t} \geq 0 .
$$

Gauss curvature flow: In fact we consider the more general class $F(W)=$ $\left(\Pi \lambda_{i}\right)^{\beta}$, where $W$ is the Weingartan map, hence $\Phi(A)=\left(\Pi \frac{1}{\mu_{i}}\right)^{\beta}$, where $\mu_{i}$ are eigenvalues of $A=W^{-1}=g^{*} \operatorname{Hess}_{\bar{\nabla}} s+s$ id. $\Psi=-\Phi=-\left(\Pi \frac{1}{\mu_{i}}\right)^{\beta}$. Using the computational lemma we have that

$$
\begin{aligned}
\ddot{\Psi}(X, X)= & \sum_{p \neq q} \frac{\beta^{2} \Psi}{\mu_{p} \mu_{q}} X_{p}^{p} X_{q}^{q}+\sum_{p} \frac{\left(\beta^{2}+\beta\right) \Psi}{\mu_{p}^{2}}\left|X_{p}^{p}\right|^{2} \\
& +\beta \Psi \sum_{p \neq q} \frac{1}{\mu_{p} \mu_{q}}\left|X_{p}^{q}\right|^{2} \\
= & \sum_{p q} \frac{\beta^{2} \Psi}{\mu_{p} \mu_{q}} X_{p}^{p} X_{q}^{q}+\sum_{p q} \frac{\beta \Psi}{\mu_{p} \mu_{q}}\left|X_{p}^{q}\right|^{2}
\end{aligned}
$$

which is negative since $\Psi<0$, and

$$
\begin{aligned}
\dot{\Psi}(X) & =\frac{\beta \Psi}{\mu_{p}} X_{p}^{p} \\
\frac{(\dot{\Psi}(X))^{2}}{\Psi} & =\beta^{2} \Psi\left(\sum \frac{1}{\mu_{p}} X_{p}^{p}\right)^{2} .
\end{aligned}
$$

Letting $Y_{p}^{p}=\frac{1}{\mu_{p}} X_{p}^{p}$ and observing

$$
\left(\sum Y_{p}^{p}\right)^{2} \leq n \sum\left|Y_{p}^{p}\right|^{2}
$$


Hence

$$
\begin{aligned}
\ddot{\Psi}(X, X) & =\frac{(\dot{\Psi}(X))^{2}}{\Psi}+\sum_{p q} \frac{\beta \Psi}{\mu_{p} \mu_{q}}\left|X_{p}^{q}\right|^{2} \\
& \leq \frac{(\dot{\Psi}(X))^{2}}{\Psi}+\sum_{p} \frac{\beta \Psi}{\mu_{p} \mu_{p}}\left|X_{p}^{p}\right|^{2} \\
& \leq \frac{(\dot{\Psi}(X))^{2}}{\Psi}+\frac{1}{n \beta} \frac{(\dot{\Psi}(X))^{2}}{\Psi} .
\end{aligned}
$$

Hence the $\beta$-power Gauss curvature flow is $-n \beta$ concave and we have Chow's

$$
\frac{\partial}{\partial t} F-h^{-1}(\nabla F, \nabla F)+\frac{n \beta F}{(1+n \beta) t} \geq 0 .
$$

Despite the generality of Theorem 2.17 the proof, which shall be given next, seems a lot easier than the ones for the special cases in $[\mathbf{H 4}, \mathbf{C h 1}]$.

C. PDE satisfied by the speed function. We shall prove Theorem 2.17 and discuss the $\frac{1}{H}$-flow case, to which Theorem 2.17 does not apply.

Start from (2.60). Take Hess $\bar{\nabla}_{\bar{\nabla}}$ on both sides and notice the commutativity of the $\frac{\partial}{\partial t}$ and $\operatorname{Hess}_{\bar{\nabla}}$ we have that

$$
\frac{\partial}{\partial t}\left(\operatorname{Hess}_{\bar{\nabla}} s+\bar{g} s\right)=\operatorname{Hess}_{\bar{\nabla}} \Psi+\Psi \bar{g} .
$$

Then

$$
\frac{\partial}{\partial t} A=\bar{g}^{*} \operatorname{Hess}_{\bar{\nabla}} \Psi+\Psi \text { id }
$$

This enables us to compute the time derivative of the speed function $\Psi$ as follows

$$
\begin{aligned}
\frac{\partial \Psi}{\partial t} & =\dot{\Psi}(A)\left[\frac{\partial A}{\partial t}\right] \\
& =\dot{\Psi}(A)\left[\bar{g}^{*} \operatorname{Hess}_{\bar{\nabla}} \Psi+\Psi \mathrm{id}\right] \\
& =\mathcal{L} \Psi+\dot{\Psi}(A)[\mathrm{id}] \Psi
\end{aligned}
$$

Here $\dot{\Psi}(A)[B]$ is the previous $\dot{\Psi}(B)$. We write this way since it is more clear to specify that $\dot{\Psi}$ is computed at $A$. We shall return to the previous notation when there is no confusion. $\mathcal{L} \varphi=\dot{\Psi}(A)\left[\bar{g}^{*} \operatorname{Hess}_{\bar{\nabla}} \varphi\right]$.

For the proof we also need the PDE on $P:=\frac{\partial}{\partial t} \Psi$. Let

$$
Q=\frac{\partial}{\partial t} A=\bar{g}^{*} \operatorname{Hess}_{\bar{\nabla}} \Psi+\Psi \mathrm{id} .
$$


Taking one more derivative on (2.71) we have that

$$
\begin{aligned}
\frac{\partial}{\partial t} P & =\ddot{\Psi}(Q, Q)+\dot{\Psi}\left(\bar{g}^{*} \operatorname{Hess}_{\bar{\nabla}} \Psi_{t}+\Psi_{t} \mathrm{id}\right) \\
& =\mathcal{L} P+\dot{\Psi}(\mathrm{id}) P+\ddot{\Psi}(Q, Q) .
\end{aligned}
$$

Note that the first equation in (2.71) is equivalent to

$$
P=\dot{\Psi}(Q) .
$$

Hence in the case of $\Psi$ being $\alpha$-concave we have that

$$
\frac{\partial}{\partial t} P \leq \mathcal{L} P+\dot{\Psi}(\mathrm{id}) P+\frac{\alpha-1}{\alpha} \frac{P^{2}}{\Psi} .
$$

Let $L=t P+\frac{\alpha}{\alpha-1} \Psi$. Using (2.71) and (2.74) we then have that

$$
\begin{aligned}
\frac{\partial}{\partial t} L \leq & \mathcal{L}(t P)+t P \dot{\Psi}(i d)+t \frac{\alpha-1}{\alpha} \frac{P^{2}}{\Psi}+P \\
& +\frac{\alpha}{\alpha-1} \mathcal{L} \Psi+\frac{\alpha}{\alpha-1} \Psi \dot{\Psi}(i d) \\
= & \mathcal{L} L+\dot{\Psi}(\mathrm{id}) L+\frac{P}{\Psi} \frac{\alpha-1}{\alpha} L .
\end{aligned}
$$

Theorem 2.17 follows from the maximum principle and the observation that $L \leq 0$ at the initial time (noting that $\Psi<0$ at $t=0$ ).

If $\alpha=1$, we are in the degenerate case in the definition of the $\alpha$-concavity. If we also have that $\Psi \geq 0$, as in the $\frac{1}{H}$-curvature flow case, we can only conclude from the above proof that both

$$
\sup _{M} \Psi_{t} \quad \text { and } \quad \sup _{M} \frac{\partial}{\partial t}(\log \Psi)=\sup _{M} \frac{\Psi_{t}}{\Psi}
$$

are monotone non-increasing.

The following calculation shows that the $\frac{1}{H}$-curvature flow is indeed 1-concave. First $\Psi=\frac{1}{\sum \frac{1}{\mu_{i}}}$, hence

$$
\frac{\partial \Psi}{\partial \mu_{i}}=\frac{\Psi^{2}}{\mu_{i}^{2}}
$$

and for $i \neq j$

$$
\frac{\partial^{2} \Psi}{\partial \mu_{i} \partial \mu_{j}}=\frac{2 \Psi^{3}}{\mu_{i}^{2} \mu_{j}^{2}},
$$

for $i=j$

$$
\frac{\partial^{2} \Psi}{\partial \mu_{i} \partial \mu_{j}}=\frac{2 \Psi^{2}}{\mu_{i}^{3}}\left(\frac{\Psi}{\mu_{i}}-1\right) .
$$


Now compute

$$
\begin{aligned}
\ddot{\Psi}(X, X)= & \sum_{p \neq q} \frac{2 \Psi^{3}}{\mu_{p}^{2} \mu_{q}^{2}} X_{p}^{p} X_{q}^{q}+\sum_{p} \frac{2 \Psi^{2}}{\mu_{p}^{3}}\left(\frac{\Psi}{\mu_{p}}-1\right)\left|X_{p}^{p}\right|^{2} \\
& -\sum_{p \neq q} \frac{\Psi^{2}\left(\mu_{p}+\mu_{q}\right)}{\mu_{p}^{2} \mu_{q}^{2}}\left|X_{p}^{q}\right|^{2} \\
= & \sum_{p, q} \frac{2 \Psi^{3}}{\mu_{p}^{2} \mu_{q}^{2}} X_{p}^{p} X_{q}^{q}-\sum_{p, q} \frac{\Psi^{2}\left(\mu_{p}+\mu_{q}\right)}{\mu_{p}^{2} \mu_{q}^{2}}\left|X_{p}^{q}\right|^{2} .
\end{aligned}
$$

On the other hand

$$
\frac{(\dot{\Psi}(X))^{2}}{\Psi}=\Psi^{3}\left(\sum \frac{1}{\mu_{p}^{2}} X_{p}^{p}\right)^{2}
$$

If we let $Y_{p}^{q}=\frac{1}{\mu_{p} \mu_{q}} X_{p}^{q}$ we have that

$$
\begin{aligned}
\ddot{\Psi}(X, X)= & 2 \Psi^{3} \sum Y_{p}^{p} Y_{q}^{q}-\Psi^{2} \sum\left(\mu_{p}+\mu_{q}\right)\left(Y_{p}^{q}\right)^{2} \\
& \leq 2 \Psi^{3}\left(\sum Y_{p}^{p}\right)^{2}-2 \Psi^{2} \sum \sqrt{\mu_{p} \mu_{q}}\left(Y_{p}^{q}\right)^{2} \\
& \leq 2 \Psi^{3}\left(\sum Y_{p}^{p}\right)^{2}-2 \Psi^{2} \sum \mu_{p}\left(Y_{p}^{p}\right)^{2} \\
& \leq 0 .
\end{aligned}
$$

Since there exists a situation that all the inequalities hold equality, we conclude that $\Psi$ is only concave.

At last, we examine the equation (2.71) for concrete examples.

Mean curvature flow: $\Psi=-\sum \frac{1}{\mu_{i}}=-H$. Hence, we have that $\mathcal{L} \varphi=$ $\frac{1}{\mu_{i}^{2}} \bar{\nabla}_{i} \bar{\nabla}_{i} \varphi=\left(W^{2}\right)^{i j} \bar{\nabla}_{i} \bar{\nabla}_{j} \varphi$. Here $W^{2}$ is the square of the Weingarten map. We abuse ${ }^{i j}$ to just mean the $(i, j)$-th component of $W^{2}$. The equation satisfied by the speed function (on sphere $\mathbb{S}^{n}$ ) is

$$
\frac{\partial}{\partial t} \Psi=\left(W^{2}\right)^{i j} \bar{\nabla}_{i} \bar{\nabla}_{j} \Psi+|W|^{2} \Psi
$$

Gauss curvature flow: $\Psi=-\frac{1}{\Pi \mu_{i}}=-K$. Hence $\mathcal{L} \varphi=(-\Psi) W^{i j} \bar{\nabla}_{i} \bar{\nabla}_{j} \varphi$. The equation satisfied by the speed function (on sphere $\mathbb{S}^{n}$ ) is

$$
\frac{\partial}{\partial t} \Psi=(-\Psi)(W)^{i j} \bar{\nabla}_{i} \bar{\nabla}_{j} \Psi-\Psi^{2} H
$$

Harmonic mean curvature flow: $\Psi=-\frac{1}{\sum \mu_{i}}, \mathcal{L} \varphi=\Psi^{2} \bar{\Delta} \varphi$. The equation satisfied by the speed function is

$$
\frac{\partial \Psi}{\partial t}=\Psi^{2} \bar{\Delta} \Psi+n \Psi^{3} .
$$


The $\frac{1}{H}$-flow: $\Psi=\frac{1}{\sum \frac{1}{\mu_{i}}}$, and $\mathcal{L} \varphi=\Psi^{2}\left(W^{2}\right)^{i j} \bar{\nabla}_{i} \bar{\nabla}_{j} \varphi$. The equation satisfied by the speed function is

$$
\frac{\partial \Psi}{\partial t}=\Psi^{2}\left(W^{2}\right)^{i j} \bar{\nabla}_{i} \bar{\nabla}_{j} \Psi+\Psi^{3}|W|^{2} .
$$

\section{Implied monotonicity}

The LYH inequality often has some immediate consequence on the monotonicity. For instance (2.2) implies that $t^{\frac{n}{2}} u(x, t)$ is monotone increasing in $t$. In the case that $h$ is closed $(1,1)$ form, $(2.17)$ concludes that

$$
\frac{\partial}{\partial t} H+\partial H(V)+\bar{\partial} H(\bar{V})+h(V, \bar{V})+\frac{H}{t} \geq 0
$$

which in turn says that $t H(x, t)$ is monotone increasing. This also applies to (2.40). Similar conclusions can be drawn from (2.18), (2.22), (2.24) etc. In this section we shall discuss those less obvious monotonicities, mostly in the integral form, implied by the LYH inequalities. They can be roughly divided into three different classes. The first type is obtained by applying the matrix LYH to submanifolds, or high order symmetric functions in stead of the trace. The second type is derived using a consideration from thermodynamics. This includes Perelman's entropy formula. The third type, which contains Hamilton's entropy formula for Ricci flow on surfaces and the Gauss curvature flow, is inferred from the long time existence and an ODE consideration. For the later two types, not the result but the proof of the LYH inequalities is needed.

3.1. Linear heat equation. Again, we present the linear heat equation due to its simplicity. Most monotonicity derived for the linear heat equation has its nonlinear analogue for Ricci flow. This is somewhat striking.

A. From the matrix LYH inequalities. It was observed first by Hamilton [H6] that Theorem 2.3 implies several previously known monotonicity formulae, including Huisken's monotonicity formula for the mean curvature flow in $\mathbb{R}^{n}$, and Struwe's monotonicity formula for the harmonic maps from Euclidean domains.

Let $(M, g)$ be as in Theorem 2.3. Let $T>0$ and $\tau=T-t$. Now let $k(x, \tau)$ be the fundamental solution to the backward heat equation:

$$
\left(\frac{\partial}{\partial \tau}-\Delta\right) k(x, \tau)=0
$$

Mean curvature flow: Let $\mathcal{V}$ be the family of submanifolds evolved by the mean curvature flow. More precisely, let $X(\cdot, t): V^{v} \rightarrow M$ be a family of embeddings ( $v$ is the dimension of $V$ ) satisfying (2.48) with $f$ being the 
mean curvature $H(x, t)$. Let $\vec{H}=H \nu$ be the mean curvature vector with $\nu$ being a unit normal. We use $D$ to denote the differentiation with respect to the metric and connection of $M$. We use $i, j, k, \cdots$ for basis of vectors tangent to $V$ and $\alpha, \beta, \gamma, \cdots$ for the basis of vectors normal to $V$. (More precisely $X(x, t)$ satisfies $\frac{\partial}{\partial t} X(x, t)=-\vec{H}(x, t)$ with $\vec{H}=\left(-\sum_{i} D_{e_{i}} e_{i}\right)^{\perp}$.) Let $H^{\alpha}=g^{i j} h_{i j}^{\alpha}$ where $h_{i j}^{\alpha}$ is the second fundamental form. We also have the following equations.

$$
\begin{aligned}
\frac{\partial}{\partial t} g_{i j} & =-2 g_{\alpha \beta} H^{\alpha} h_{i j}^{\beta}, \\
\frac{\partial}{\partial t} d \mu & =-|H|^{2} d \mu, \\
\Delta_{M} k & =\Delta_{V} k+g^{\alpha \beta} D_{\alpha} D_{\beta} k+\langle\vec{H}, D k\rangle .
\end{aligned}
$$

With respect to the evolving metrics, there exists the heat operator on $V$. It is easy to compute that for $u$ defined on $M$, the following is the conjugate heat equation:

$$
\Delta_{V} u-u_{\tau}-\langle\vec{H}, D u\rangle-|H|^{2} u=0 .
$$

By Theorem 2.3, we have that

$$
\Delta_{V} u-u_{\tau}-\langle\vec{H}, D u\rangle-|H|^{2} u=-\tau^{\frac{n-v}{2}}\left|\vec{H}+D^{\perp} \log k\right|^{2} k .
$$

for $u=\tau^{\frac{n-v}{2}} k$. It immediately implies the following result.

Theorem 3.1. Let

$$
I(t)=\tau^{\frac{n-v}{2}} \int_{V} k(x, \tau) d \mu_{t} .
$$

Then

$$
\frac{d}{d t} I(t)=-\tau^{\frac{n-v}{2}} \int k\left|\vec{H}+D^{\perp} \log k\right|^{2} d \mu_{t} .
$$

Harmonic map heat flow: Let $F(\cdot, t)$ be a family of maps from $M$ into another Riemannian manifold $N$ satisfying that harmonic map heat equation

$$
\frac{\partial}{\partial t} F=\Delta F
$$

where locally

$$
(\Delta F)^{\alpha}=g^{i j}\left(\frac{\partial^{2} F}{\partial x^{i} \partial x^{j}}-\Gamma_{i j}^{k} \frac{\partial F^{\alpha}}{\partial x^{k}}+\bar{\Gamma}_{\beta \gamma}^{\alpha}(F) \frac{\partial F^{\beta}}{\partial x^{i}} \frac{\partial F^{\gamma}}{\partial x^{j}}\right) .
$$


THEOREM 3.2. Let $F$ be a solution to the harmonic map heat flow and let

$$
I(t)=\tau \int_{M}|D F|^{2} k d \mu
$$

The following identity holds on any $M$ :

$$
\frac{d}{d t} I(t)=-2 \tau \int_{M}\left(\left|\Delta F+\frac{D k}{k} \cdot D F\right|^{2}+\left(D_{i} D_{j} \log k+\frac{1}{2 \tau} g_{i j}\right) D_{i} F D_{j} F\right) k .
$$

In particular if $(M, g)$ is as in Theorem 2.3 then $I(t)$ is non-increasing in $t$.

The proof is direct computation and integration by parts. The key identities are

$$
\begin{aligned}
\int_{M} \Delta k|D F|^{2} & =-2 \int_{M} D_{j} k D_{j} D_{i} F^{\alpha} D_{i} F^{\alpha} \\
\int_{M} D_{i} D_{j} k D_{i} F^{\alpha} D_{j} F^{\alpha} & =-\int_{M} \Delta F^{\alpha} D_{j} F^{\alpha} D_{j} k+\frac{1}{2} \int_{M} \Delta k|D F|^{2} .
\end{aligned}
$$

Yang-Mills flow: This is similar to the harmonic map heat flow. Now the equation is on a family of connections $A=\left(A_{j \beta}^{\alpha}\right)$ on a vector bundle $E$. Let $F_{A}=F_{i j \beta}^{\alpha}$ be the curvature of $A$. The Yang-Mills equation is

$$
\frac{\partial}{\partial t} A=\operatorname{div} F_{A}
$$

where $\operatorname{div} F_{k \beta}^{\alpha}=g^{i j} D_{i} F_{j k \beta}^{\alpha}$.

THEOREM 3.3. Let

Then

$$
I(t)=\tau^{2} \int_{M}|F|^{2} k
$$

$$
\begin{aligned}
\frac{d}{d t} I(t)= & -4 \tau^{2} \int_{M}\left(\left(D_{i} D_{j} \log k+\frac{1}{2 \tau} g_{i j}\right) F_{i k \beta}^{\alpha} F_{i k \beta}^{\alpha}\right. \\
& \left.+\left|\operatorname{div} F_{j \beta}^{\alpha}+D_{i} \log k F_{i j \beta}^{\alpha}\right|^{2}\right) k
\end{aligned}
$$

Kähler case: Applying Theorem 2.4 and Theorem 2.5 one can obtain results similar as the above. Due to the fact that the estimate now is on complex Hessian only, the results are on holomorphic objects. The first result is in the same line as Theorem 3.1, but with the opposite monotonicity since we apply (2.4) to the heat kernel instead of the fundamental solution to the backward heat equation.

ThEOREM 3.4. Let $M$ be a complete Kähler manifold with nonnegative bisectional curvature. Let $H(x, t ; y, 0)$ be the fundamental solution of the 
heat equation. Let $\mathcal{V} \subset M$ be any complex subvariety of dimension s. Let $K_{\mathcal{V}}(x, t ; y, 0)$ be the fundamental solution of heat equation on $\mathcal{V}$. Then

(i)

$$
K_{\mathcal{V}}(x, t ; y, 0) \leq(\pi t)^{m-s} H(x, t ; y, 0) \text {, for any } x, y \in \mathcal{V}
$$

If the equality holds, then $\mathcal{V}$ is totally geodesic. Furthermore if $\tilde{M}$ is the universal cover of $M$ with covering map $\pi$ and $\tilde{\mathcal{V}}=\pi^{-1}(\mathcal{V})$, then $\tilde{M}=$ $\tilde{M}_{1} \times \mathbb{C}^{k}$ for some Kähler manifold $\tilde{M}_{1}$ which does not contain any Euclidean factors, with $k \geq m-s$. Moreover $\tilde{\mathcal{V}}=\tilde{M}_{1} \times \mathbb{C}^{l}$ with $l<k$.

(ii)

$$
\frac{d}{d t} \int_{\mathcal{V}}(\pi t)^{m-s} H(x, t ; y, 0) d A_{\mathcal{V}}(y) \geq 0, \text { for any } x \in M .
$$

Similarly, if the equality holds for some $x \in M$ at some positive time $t$, then $\tilde{M}=\tilde{M}_{1} \times \mathbb{C}^{k}$ with $k \geq m-s$.

The result has applications in obtaining the sharp dimension estimates on the space of holomorphic functions with polynomial growth [N3].

Theorem 2.4 also implies the monotonicity of the weighted energy of a holomorphic mapping from $M$ (into any Kähler manifolds), as well as the monotonicity of the weighted energy for Hermitian-Einstein flow on any holomorphic vector bundle over $M$.

Let $F$ be a holomorphic mapping from $M$ (into, say, another Kähler manifold $N$ ), then we have that

$$
\frac{d}{d t}\left((T-t) \int_{M}|\partial F|^{2} k\left(x, t ; x_{0}, T\right) d \mu\right) \leq 0
$$

where $|\partial F|^{2}=g^{\alpha \bar{\beta}} h_{i \bar{j}} F_{\alpha}^{i} F_{\bar{\beta}}^{\bar{j}}, k\left(x, t ; x_{0}, T\right)$ is the fundamental solution to the backward heat equation satisfying $\left(\frac{\partial}{\partial t}+\Delta\right) k\left(x, t ; x_{0}, T\right)=\delta_{\left(x_{0}, T\right)}(x, t)$. Dually we also have that

$$
\frac{d}{d t}\left(t \int_{M}|\partial F|^{2} H\left(x, t ; x_{0}, 0\right) d \mu\right) \geq 0
$$

where $H\left(x, t ; x_{0}, 0\right)$ is the fundamental solution of the heat equation centered at $\left(x_{0}, 0\right)$.

Let $H(t)$ be a family of metrics of a holomorphic vector bundle $E$ satisfying the Hermitian-Einstein equation

$$
\frac{\partial h}{\partial t} h^{-1}=-\Lambda F+\lambda \mathrm{I}
$$

where $h_{\alpha}^{\beta}(t)=H(t)_{\alpha \bar{\gamma}} H(0)^{\bar{\gamma} \beta}$, a Hermitian symmetric morphism of $E$. Then

$$
\frac{d}{d t}\left((T-t)^{2} \int_{M}|F|^{2} k\left(x, t ; x_{0}, T\right) d \mu\right) \leq 0
$$


where $|F|^{2}=\left|F_{i \bar{j} \alpha^{\beta}}\right|^{2}$.

B. Thermodynamical consideration. In the thermodynamics the concept of the entropy is used to characterize the equilibrium states. In this setting, there is entropy function $S$ which depends on state variable energy $E$ as well as other parameters. The following are assumed: The entropy function $S$ and energy $E$ satisfy

1) $\frac{\partial S}{\partial E}>0$ (hence define $\frac{1}{\tau} \doteqdot \frac{\partial S}{\partial E}$, with $\tau$ being interpreted as the temperature);

2) $S$ is concave in $E$;

3) $S$ is positively homogenous of degree 1 .

Written as functions of $S$, there is free energy $F \doteqdot E-T S$.

In statistical mechanics, the entropy was used to measure the uncertainty. Here the equilibrium is characterized as the distribution maximizing the entropy $(=$ uncertainty $)$. Let $(M, d \mu)$ be a manifold with measure. Let $H:(M, d \mu) \rightarrow \mathbb{R}$ be the Hamiltonian. We have the following definitions.

Partition function: $Z:=\int_{M} e^{-\beta H} d \mu$;

Temperature: $\tau:=\frac{1}{\beta}$;

Energy: $E:=-\frac{\partial}{\partial \beta} \log Z \geq 0$;

Entropy: $S:=\beta E+\log Z$;

Free energy: $F:=-\frac{1}{\beta} \log Z$.

The following result recovers the aspects of the classical equilibrium thermodynamics.

THEOREM 3.5.

$$
\begin{aligned}
E & =\langle H\rangle, \text { with respect to the canonical distribution } \sigma=\frac{1}{Z} e^{-\beta H}, \\
S & =S(\sigma)=-\int_{M} \sigma \log \sigma d \mu, \\
\frac{\partial S}{\partial E} & =\frac{1}{\tau}, \quad F=E-S \tau, \quad \frac{\partial F}{\partial \tau}=-S .
\end{aligned}
$$

Define the heat capacity

$$
C_{V}=\frac{\partial E}{\partial \tau}=-\beta^{2} \frac{\partial E}{\partial \beta} .
$$

Corollary 3.6. $C_{V} \geq 0$ and

$$
\begin{aligned}
C_{V} & =\beta^{2} \frac{\partial^{2}}{\partial \beta^{2}} \log Z, \\
\frac{\partial^{2} S}{\partial E^{2}} & =\left(\frac{\partial E}{\partial \beta}\right)^{-1} \leq 0, \\
\frac{\partial S}{\partial \beta} & =\beta \frac{\partial E}{\partial \beta} \leq 0 .
\end{aligned}
$$


This consideration suggests the following entropy formula. First after (2.4) (replacing $t$ by $\tau$ ) denote that $c_{v}=\frac{2}{u}\left|\Upsilon_{i j}\right|^{2}+\frac{2}{u} R_{i j} \nabla_{i} u \nabla_{j} u$. Then

$$
\frac{\partial}{\partial \tau} \int_{M} Q d \mu=\int_{M} c_{v} d \mu-\frac{2}{\tau} \int_{M} Q d \mu .
$$

Equivalently,

$$
\frac{\partial}{\partial \tau}\left(\tau^{2} \int_{M} Q d \mu\right)=C_{V}
$$

where $C_{V}=\tau^{2} \int_{M} c_{v} d \mu$. This suggests that $\tau^{2} \int_{M} Q$ should be the energy $E$. Normalizing $\int_{M} u d \mu=1$, then $\log Z$ (essentially it is the anti-derivative of $E$ in terms of $\frac{1}{\tau}$ ) can be computed.

$$
\log Z=\int_{M} u \log u+\frac{n}{2} \log (4 \pi \tau)+\frac{n}{2} .
$$

This gives the entropy

$$
S=\int_{M}(-\tau \Delta f-f+n) u d \mu=-\int_{M}\left(\tau|\nabla f|^{2}+f-n\right) u d \mu .
$$

The following is a direct consequence of Corollary 3.6.

Theorem 3.7. Let $W=-S$. Then

$$
\frac{d \mathcal{W}}{d \tau}=-2 \tau \int_{M}\left(\left|\nabla_{i} \nabla_{j} f-\frac{1}{2 \tau} g_{i j}\right|^{2}+R_{i j} f_{i} f_{j}\right) u d \mu .
$$

In particular, if $M$ has nonnegative Ricci curvature, $\mathcal{W}(f, \tau)$ is monotone decreasing along the heat equation.

3.2. Ricci flow. The most important monotonicity formula for the Ricci flow is the entropy monotonicity discovered by Perelman $[\mathbf{P}]$. However, before this spectacular result, several monotonicity results have been obtained by Hamilton, mostly for surfaces, including his entropy and isoperimetric constant monotonicity.

A. Hamilton's entropy for surfaces. In [H2], Hamilton discovered an entropy monotonicity for the Ricci flow on the Riemann sphere with positive curvature. It is derived out of the proof for the LYH inequality for the surface case and the long time existence for the normalized flow: $\frac{\partial}{\partial t} g_{i j}=(r-R) g_{i j}$, where $r=\frac{\int R d \mu_{t}}{A\left(M_{t}\right)}$ is the average of $R$. It is easy to see that the normalized flow preserves the area $A\left(M_{t}\right)$. Also it is not hard to show that the normalized flow has a long time solution $[\mathbf{H 2}]$.

Theorem 3.8. Let $I(t)=\int_{M} R \log R$. Then $I(t)$ is monotone nonincreasing in $t$. 
Direct computation, using $\left(\frac{\partial}{\partial t}-\Delta\right) R=R^{2}-r R$, shows that

$$
\frac{d}{d t} I(t)=\int_{M}(\Delta R) \log R+R^{2}-R r=\int_{M}(\Delta \log R+R-r) R .
$$

Let $Q=R(\Delta \log R+R-r)$. Similar as (2.4) we have that

$$
\left(\frac{\partial}{\partial t}-\Delta\right) Q=2\left|\nabla_{i} \nabla_{j} \log R+\frac{1}{2}(R-r) g_{i j}\right|^{2} R+r Q+Q(R-r)
$$

which implies Hamilton's LYH inequality for surfaces by applying maximum principle. Integrating the above one has that

$$
\frac{d}{d t} \int_{M} Q d \mu=\int_{M} 2\left|\nabla_{i} \nabla_{j} \log R+\frac{1}{2}(R-r) g_{i j}\right|^{2} R+r \int_{M} Q
$$

Using

$$
\left(\int_{M} Q\right)^{2} \leq\left(\int_{M}\left(\frac{Q}{R}\right)^{2} R\right) \int_{M} R \leq 8 \pi \int_{M} 2\left|\nabla_{i} \nabla_{j} \log R+\frac{1}{2}(R-r) g_{i j}\right|^{2} R
$$

the above implies that if denote $Z=\int_{M} Q d \mu=I^{\prime}$,

$$
Z^{\prime} \geq \frac{1}{8 \pi} Z^{2}+r Z
$$

By ODE comparison we conclude that $Z \leq 0$, otherwise $Z$ has to blow up at some finite time.

It is easy to see from the proof that if $Z=0$ for some $t_{0},(M, g)$ is a gradient shrinking soliton.

B. From matrix LYH inequalities. The first result is a consequence of Cao's inequality (2.22). A simple consequence is the following trace inequality:

$$
\frac{\partial R}{\partial t}+X_{s} \nabla_{\bar{s}} R+X_{\bar{s}} \nabla_{s} R+R_{i \bar{j}} X_{\bar{i}} X_{j}+\frac{R}{t} \geq 0
$$

In particular, we have that

$$
\frac{\partial R}{\partial t}-\frac{|\nabla R|^{2}}{R}+\frac{R}{t} \geq 0 .
$$

In $[\mathbf{C o}]$, the following result was derived on $S_{n}(x, t):=\frac{\operatorname{det}\left(R_{i \bar{j}}\right)}{\operatorname{det}\left(g_{i j}\right)}$, the $n$-th symmetric function of Ric with respect to the Kähler metric $\omega$,

$$
\frac{\partial S_{n}}{\partial t}-\frac{\left|\nabla S_{n}\right|^{2}}{n S_{n}}+\frac{n S_{n}}{t} \geq 0
$$


We shall derive the similar results for general symmetric functions of Ric with respect to $\omega$.

First we have to set some notations. Here we follow $[\mathbf{H S}]$ (see also $[\mathbf{A n} \mathbf{1}]$ ). Let $A$ be a symmetric (Hermitian symmetric) matrix. Denote by $\lambda_{i}$ its eigenvalues.

$$
S_{k}(\lambda):=\sum_{\alpha_{1}<\alpha_{2}<\cdots<\alpha_{k}} \lambda_{\alpha_{1}} \lambda_{\alpha_{2}} \cdots \lambda_{\alpha_{k}}
$$

The symmetric functions $S_{k}(\lambda)$ can also be viewed as functions of matrix $A$ (when so we write as $S_{k}(A)$ ). Define the map

$$
A^{(k)}\left(e_{\alpha_{1}} \otimes \cdots e_{\alpha_{k}}\right):=\frac{1}{k !} \sum_{\sigma \in S(k)}(-1)^{\operatorname{sgn}(\sigma)} A\left(e_{\sigma\left(\alpha_{1}\right)}\right) \otimes \cdots A\left(e_{\sigma\left(\alpha_{k}\right)}\right) .
$$

Then we have that $S_{k}(\lambda)=\operatorname{tr} A^{(k)}$. Now it is easy to show that at the points where $A$ is diagonal we have that

$$
\left(\frac{\partial S_{k}(A)}{\partial a_{i j}}\right)=\operatorname{diag}\left(\frac{\partial S_{k}(\lambda)}{\partial \lambda_{1}}, \cdots, \frac{\partial S_{k}(\lambda)}{\partial \lambda_{n}}\right)
$$

It is also easy to see that

$$
S_{k}(\text { Ric })=\frac{n !}{k !(n-k) !} \frac{\operatorname{Ric}^{k} \wedge \omega^{n-k}}{\omega^{n}} .
$$

Let $S_{k, i}(\lambda)$ be the sum of terms of $S_{k}(\lambda)$ not containing the factor $\lambda_{i}$. We shall need the following identities, which are proved in $[\mathbf{H S}]$.

LEMMA 3.9.

$$
\begin{aligned}
\frac{\partial S_{k}(\lambda)}{\partial \lambda_{i}}(\lambda) & =S_{k-1, i}(\lambda), \\
S_{k}(\lambda) & =S_{k, i}(\lambda)+\lambda_{i} S_{k-1, i}(\lambda), \\
\sum_{i=1}^{n} S_{k, i}(\lambda) & =(n-k) S_{k}(\lambda), \\
\sum_{i=1}^{n} \lambda_{i} S_{k-1, i}(\lambda) & =k S_{k}(\lambda), \\
\sum_{i=1}^{n} \lambda_{i}^{2} S_{k-1, i}(\lambda) & =S_{1}(\lambda) S_{k}(\lambda)-(k+1) S_{k+1}(\lambda) .
\end{aligned}
$$

Armed with the above, we shall first compute $\frac{\partial}{\partial t}\left(\log S_{k}(\mathrm{Ric})\right)$. (In the following we shall simply denote $S_{k}(\mathrm{Ric})$ by $S_{k}(x, t), S_{k}$, or $S_{k}(\lambda)$, where $\lambda_{i}$ are eigenvalues of Ric with respect to $\omega)$. 
LEMMA 3.10. With respect to a unitary frame such that Ric is diagonal we have

$$
\begin{gathered}
\frac{\partial}{\partial t}\left(\log S_{k}(\lambda)\right)=\frac{1}{S_{k}(\lambda)}\left(\sum_{i=1}^{n} S_{k-1, i}(\lambda) \frac{\partial}{\partial t} R_{i \bar{i}}-(k+1) S_{k+1}(\lambda)\right)+S_{1}(\lambda) \\
\nabla_{s}\left(\log S_{k}(\lambda)\right)=\frac{\sum_{i} \nabla_{s} R_{i \bar{i}} S_{k-1, i}(\lambda)}{S_{k}(\lambda)} .
\end{gathered}
$$

ProOF. First by the direct calculation we have that

$$
\frac{\partial}{\partial t}\left(\log S_{k}(\lambda)\right)=\frac{k \operatorname{Ric}^{k-1} \wedge \frac{\partial}{\partial t} \operatorname{Ric} \wedge \omega^{n-k}-(n-k) \operatorname{Ric}^{k+1} \wedge \omega^{n-k-1}}{\operatorname{Ric}^{k} \wedge \omega^{n-k}}+S_{1}(\lambda)
$$

Direct calculation shows that

$$
k \operatorname{Ric}^{k-1} \wedge \frac{\partial}{\partial t} \operatorname{Ric} \wedge \omega^{n-k}=\frac{k !(n-k) !}{n !} \sum_{i=1}^{n}\left(\frac{\partial}{\partial t} R_{i \bar{i}}\right) S_{k-1, i}(\lambda) \omega^{n}
$$

and

$$
(n-k) \operatorname{Ric}^{k+1} \wedge \omega^{n-k-1}=\frac{(k+1) !(n-k) !}{n !} S_{k+1}(\lambda) \omega^{n} .
$$

Putting them together we prove (3.26). The proof of (3.27) follows from (3.20) and (3.21).

THEOREM 3.11. Under the assumption that $(M, g(t))$ is a solution to the Kähler-Ricci flow with bounded nonnegative bisectional curvature, we have that

$$
\frac{\partial S_{k}}{\partial t}-\frac{1}{k} \frac{\left|\nabla S_{k}\right|^{2}}{S_{k}}+\frac{k}{t} S_{k} \geq 0
$$

Proof. Choose a unitary frame so that Ric is diagonal. By the above lemma and (2.22) we have that

$$
\begin{aligned}
& \frac{\partial}{\partial t}\left(\log S_{k}(\lambda)\right) \\
& \quad \geq \frac{1}{S_{k}(\lambda)} \sum_{i=1}^{n} S_{k-1, i}(\lambda)\left(-\lambda_{i}^{2}-\frac{\lambda_{i}}{t}-X_{s} \nabla_{\bar{s}} R_{i \bar{i}}-X_{\bar{s}} \nabla_{s} R_{i \bar{i}}-R_{\bar{i} \bar{s} \bar{t}} X_{\bar{s}} X_{t}\right) \\
& \quad-\frac{(k+1) S_{k+1}(\lambda)}{S_{k}(\lambda)}+S_{1}(\lambda) .
\end{aligned}
$$


Using (3.25), (3.24) and (3.27) we have that

$$
\begin{aligned}
\frac{\partial}{\partial t}\left(\log S_{k}(\lambda)\right) \geq & \frac{1}{S_{k}(\lambda)}\left(-S_{1}(\lambda) S_{k}(\lambda)+(k+1) S_{k+1}(\lambda)-\frac{k S_{k}(\lambda)}{t}\right) \\
& -X_{s} \nabla_{\bar{s}} \log S_{k}(\lambda)-X_{\bar{s}} \nabla_{s} \log S_{k} \\
& -\frac{1}{S_{k}(\lambda)} \sum_{i=1}^{n} S_{k-1, i}(\lambda) R_{i \bar{i} s \bar{t}} X_{\bar{s}} X_{t}-\frac{(k+1) S_{k+1}(\lambda)}{S_{k}(\lambda)}+S_{1}(\lambda) \\
= & -\frac{k}{t}-X_{s} \nabla_{\bar{s}} \log S_{k}(\lambda)-X_{\bar{s}} \nabla_{s} \log S_{k}(\lambda) \\
& -\frac{1}{S_{k}(\lambda)} \sum_{i=1}^{n} S_{k-1, i}(\lambda) R_{i \bar{i} s \bar{t}} X_{\bar{s}} X_{t} .
\end{aligned}
$$

Notice that

$$
R_{i \bar{i} \bar{s} \bar{t}} X_{\bar{s}} X_{t}=R(i, \bar{i}, X, \bar{X})=|X|^{2} R\left(i, \bar{i}, \frac{X}{|X|}, \frac{\bar{X}}{|X|}\right) \leq|X|^{2} R_{i \bar{i}} .
$$

Hence

$$
\frac{\partial}{\partial t} \log S_{k}(\lambda) \geq-\frac{k}{t}-X_{s} \nabla_{\bar{s}} \log S_{k}(\lambda)-X_{\bar{s}} \nabla_{s} \log S_{k}(\lambda)-k|X|^{2} .
$$

Here we have used (3.24) again. The claimed result follows by choosing $X=-\frac{1}{k} \nabla \log S_{k}(\lambda)$.

The theorem in particular implies that $t^{k} S_{k}(x, t)$ is monotone non-decreasing. As in the linear case, one can also apply the matrix LYH inequality (2.29) to subvarieties of $M$.

THEOREM 3.12. Let $M$ be a complete Kähler manifold with bounded nonnegative bisectional curvature. Let $H(x, t ; y, 0)$ be a fundamental solution to the forward conjugate heat equation on $M$. Let $\mathcal{V}$ be a complex subvariety of $M$ of dimension $s$. Let $K \mathcal{V}(x, t ; y, 0)$ be the fundamental solution to the restricted forward conjugate heat equation (with respect to the induced metrics) on $\mathcal{V}$. Then we have (3.9) and (3.10). Moreover, the equality (for positive $t$ ), in either cases, implies that the universal cover (of $M$ ) $\tilde{M}$ has the splitting $\tilde{M}=\tilde{M}_{1} \times \mathbb{E}^{k}$, where $\mathbb{E}^{k}$ is a gradient expanding Kähler-Ricci soliton of dimension $k \geq m-s$.

Remark 3.13. One can think of (3.10) as a dual version of Perelman's monotonicity of the reduced volume since the reduced volume in the Section 7 of $[\mathbf{P}]$ is, in a sense, a 'weighted volume' of $M$ (with weight being the fundamental solution (to the backward conjugate heat equation) of a 'potentially infinity dimensional manifold' restricted to $M$, as explained in Section 6 of $[\mathbf{P}]$ ), while here the monotonicity is on the 'weighted volume' of complex submanifolds with weight being the fundamental solution (of the 
forward conjugate heat equation) of $M$ restricted to the submanifold. The reduced volume monotonicity of Perelman has important applications in the study of Ricci flow. We expect that (3.10) will have some applications in understanding the relation between Kähler-Ricci flow and the complex geometry of analytic subvarieties.

C. Perelman's entropy. Perelman's entropy and its monotonicity can be obtained from the thermodynamical consideration as before from (2.45). Recall that $(M, g(t))$ is a solution to the Ricci flow on $M \times[0, T]$. Let $\tau=$ $T-t$, and consider the positive solution $u$ to the conjugate heat equation

$$
\frac{\partial u}{\partial \tau}-\Delta u+R u=0
$$

Assume $\int_{M} u=1$, Integrating (2.45) we have that

$$
\frac{\partial}{\partial \tau} \int_{M} v_{0}=-2 \int_{M}\left|R_{i j}+f_{i j}\right|^{2} d \mu
$$

Using ODE, it is easy to see that $\int_{M} Q d \mu \geq 0$ if $Q=\frac{n}{2 \tau} u-v_{0}$.) Then

$$
\frac{\partial}{\partial \tau} \int_{M} Q=\int_{M} c_{v}-\frac{2}{\tau} \int_{M} Q
$$

with

$$
c_{v}=2\left|R_{i j}+f_{i j}-\frac{1}{2 \tau} g_{i j}\right|^{2} u .
$$

Exactly as the earlier linear case we let

$$
E=\tau^{2} \int_{M} Q d \mu
$$

be the energy and let $\tau$ be the temperature, then

$$
\log Z=\int_{M} u \log u+\frac{n}{2} \log (4 \pi \tau)+\frac{n}{2}
$$

obtained as the anti-derivative of $E$.

Now Corollary 3.6 implies the following entropy formula.

TheOREM 3.14. Let

$$
\mathcal{W}(g, u, \tau):=\int_{M}\left(\tau\left(|\nabla f|^{2}+R\right)+f-n\right) u d \mu .
$$

Then

$$
\frac{d \mathcal{W}}{d \tau}=-2 \tau \int_{M}\left|\nabla_{i} \nabla_{j} f+R_{i j}-\frac{1}{2 \tau} g_{i j}\right|^{2} u d \mu \leq 0 .
$$


3.3. Interpolation and localization. The interpolation we mean here is the one between the LYH inequality (2.29) and Perelman's entropy monotonicity (3.31), which we found a little mysterious. This seems to only work for Kähler cases.

Consider the Kähler-Ricci flow:

$$
\frac{\partial}{\partial \tau} g_{\alpha \bar{\beta}}=\epsilon R_{\alpha \bar{\beta}}
$$

where $\epsilon$ is a parameter and the conjugate heat equation:

$$
\left(\frac{\partial}{\partial \tau}-\Delta+\epsilon \mathcal{R}\right) u(x, \tau)=0 .
$$

When $\epsilon<0,(3.32)$ is a forward Ricci flow equation and (3.33) becomes the forward conjugate heat equation. The equations look different from those in Section 2 since in this section the case of $\epsilon<0$ corresponds to the forward Ricci flow and the case of $\epsilon>0$ corresponds to the backward Ricci flow. For example $\epsilon=1$ is exactly the setting for Perelman's entropy and energy monotonicity. Notice that (3.33) becomes the backward conjugate heat equation for $\epsilon=1$. For the positive solution $u(x, \tau)$ we define the $(1,1)$ tensor $Z_{\alpha \bar{\beta}}$ by

$$
Z_{\alpha \bar{\beta}}=-(\log u)_{\alpha \bar{\beta}}+\epsilon R_{\alpha \bar{\beta}} \text {. }
$$

Let $\Delta_{L}$ denote the Lichnerowicz Laplacian on $(1,1)$ tensors. Computation yields

$$
\begin{aligned}
\left(\frac{\partial}{\partial \tau}\right. & \left.-\Delta_{L}\right) Z_{\alpha \bar{\beta}} \\
= & -\epsilon^{2}\left(\Delta R_{\alpha \bar{\beta}}+R_{\alpha \bar{\beta} \gamma \bar{\delta}} R_{\bar{\gamma} \delta}+\nabla_{\gamma} R_{\alpha \bar{\beta}}\left(\frac{1}{\epsilon} \nabla_{\bar{\gamma}} \log u\right)\right. \\
& \left.+\nabla_{\bar{\gamma}} R_{\alpha \bar{\beta}}\left(\frac{1}{\epsilon} \nabla_{\gamma} \log u\right)+R_{\alpha \bar{\beta} \gamma \bar{\delta}}\left(\frac{1}{\epsilon} \nabla_{\bar{\gamma}} \log u\right)\left(\frac{1}{\epsilon} \nabla_{\delta} \log u\right)\right) \\
& -(\log u)_{\alpha \gamma}(\log u)_{\bar{\gamma} \bar{\beta}}+\nabla_{\gamma}\left(Z_{\alpha \bar{\beta}}\right) \nabla_{\bar{\gamma}} \log u+\nabla_{\bar{\gamma}}\left(Z_{\alpha \bar{\beta}}\right) \nabla_{\gamma} \log u \\
& +\frac{1}{2} Z_{\alpha \bar{\gamma}}\left(\epsilon R_{\gamma \bar{\beta}}+(\log u)_{\gamma \bar{\beta}}\right)+\frac{1}{2}\left(\epsilon R_{\alpha \bar{\gamma}}+(\log u)_{\alpha \bar{\gamma}}\right) Z_{\gamma \bar{\beta}} .
\end{aligned}
$$

Let

and

$$
\tilde{Z}_{\alpha \bar{\beta}}=Z_{\alpha \bar{\beta}}-\frac{1}{\tau} g_{\alpha \bar{\beta}}
$$

$$
\begin{aligned}
Y_{\alpha \bar{\beta}}= & \Delta R_{\alpha \bar{\beta}}+R_{\alpha \bar{\beta} \gamma \bar{\delta}} R_{\bar{\gamma} \delta}+\nabla_{\gamma} R_{\alpha \bar{\beta}}\left(\frac{1}{\epsilon} \nabla_{\bar{\gamma}} \log u\right)+\nabla_{\bar{\gamma}} R_{\alpha \bar{\beta}}\left(\frac{1}{\epsilon} \nabla_{\gamma} \log u\right) \\
& +R_{\alpha \bar{\beta} \gamma \bar{\delta}}\left(\frac{1}{\epsilon} \nabla_{\bar{\gamma}} \log u\right)\left(\frac{1}{\epsilon} \nabla_{\delta} \log u\right) .
\end{aligned}
$$


Notice that $\tilde{Y}_{\alpha \bar{\beta}}$ in Theorem 2.7 is related to $Y_{\alpha \bar{\beta}}$ by $\tilde{Y}_{\alpha \bar{\beta}}=Y_{\alpha \bar{\beta}}-\frac{R_{\alpha \bar{\beta}}}{\epsilon \tau}$. From (3.34), we can derive the equation for $\tilde{Z}_{\alpha \bar{\beta}}$ as follows. In terms of $\tilde{Z}$ we have that

$$
\begin{aligned}
\left(\frac{\partial}{\partial \tau}-\Delta_{L}\right) \tilde{Z}_{\alpha \bar{\beta}}= & \left(\frac{\partial}{\partial \tau}-\Delta_{L}\right) Z_{\alpha \bar{\beta}}+\frac{1}{\tau^{2}} g_{\alpha \bar{\beta}}-\frac{1}{\tau} \epsilon R_{\alpha \bar{\beta}} \\
= & -\epsilon^{2} \tilde{Y}_{\alpha \bar{\beta}}-(\log u)_{\alpha \gamma}(\log u)_{\bar{\gamma} \bar{\beta}} \\
& +\nabla_{\gamma}\left(\tilde{Z}_{\alpha \bar{\beta}}\right) \nabla_{\bar{\gamma}} \log u+\nabla_{\bar{\gamma}}\left(\tilde{Z}_{\alpha \bar{\beta}}\right) \nabla_{\gamma} \log u \\
& +\frac{1}{2} \tilde{Z}_{\alpha \bar{\gamma}}\left(\epsilon R_{\gamma \bar{\beta}}+(\log u)_{\gamma \bar{\beta}}-\frac{1}{\tau} g_{\gamma \bar{\beta}}\right) \\
& +\frac{1}{2}\left(\epsilon R_{\alpha \bar{\gamma}}+(\log u)_{\alpha \bar{\gamma}}-\frac{1}{\tau} g_{\alpha \bar{\gamma}}\right) \tilde{Z}_{\gamma \bar{\beta}}
\end{aligned}
$$

For $\epsilon<0$, applying Theorem 2.7 we know that $\epsilon^{2} Y_{\alpha \bar{\beta}}-\frac{\epsilon}{\tau} R_{\alpha \bar{\beta}} \geq 0$ under the assumption that $M$ is a complete Kähler manifold with bounded nonnegative holomorphic bisectional curvature. Hence the tensor maximum principle and (3.35) imply that $\tilde{Z}_{\alpha \bar{\beta}} \leq 0$.

Let $f=-\log u, Z=g^{\alpha \bar{\beta}} Z_{\alpha \bar{\beta}}$. Tracing (3.34) gives

$$
\begin{aligned}
\left(\frac{\partial}{\partial \tau}-\Delta\right) Z= & -\epsilon R_{\bar{\alpha} \beta} Z_{\alpha \bar{\beta}}-\epsilon^{2} g^{\alpha \bar{\beta}} Y_{\alpha \bar{\beta}}-(f)_{\alpha \gamma}(f)_{\bar{\gamma} \bar{\alpha}} \\
& -\nabla_{\gamma} Z \nabla_{\bar{\gamma}} f-\nabla_{\bar{\gamma}} Z \nabla_{\gamma} f+Z_{\alpha \bar{\beta}}\left(\epsilon R_{\beta \bar{\alpha}}-f_{\beta \bar{\alpha}}\right)
\end{aligned}
$$

and

$$
\begin{aligned}
g^{\alpha \bar{\beta}} Y_{\alpha \bar{\beta}}= & \Delta \mathcal{R}+R_{\alpha \bar{\beta}} R_{\bar{\alpha} \beta}-\nabla_{\gamma} \mathcal{R}\left(\frac{1}{\epsilon} \nabla_{\bar{\gamma}} f\right) \\
& -\left(\frac{1}{\epsilon} \nabla_{\gamma} f\right) \nabla_{\bar{\gamma}} \mathcal{R}+R_{\alpha \bar{\beta}}\left(\frac{1}{\epsilon} f_{\bar{\alpha}}\right)\left(\frac{1}{\epsilon} f_{\beta}\right) .
\end{aligned}
$$

For $\epsilon=1$, integration by parts as before gives

$$
\frac{d}{d \tau} \int_{M} \tilde{Z} u d \mu_{\tau}=-\int_{M}\left(\left|\tilde{Z}_{\alpha \bar{\beta}}\right|^{2}+\left|f_{\alpha \beta}\right|^{2}\right) u d \mu_{\tau}-\frac{2}{\tau} \int_{M} \tilde{Z} u d \mu_{\tau} .
$$

The above equation is equivalent to Perelman's entropy monotonicity since the quantity $Q$ in Perelman's entropy formula derivation above is nothing but $-\tilde{Z} u$ (for the Kähler case, there exists a factor 2 difference).

Note that for $\epsilon=-1$, the scalar curvature $R$ does satisfies (3.33) in the case $m=1$. And renormalization of (3.35) gives the previous Hamilton's result (3.16). Hence the matrix computation above also implies the Hamilton's entropy monotonicity, Theorem 3.8. It is interesting to see if (3.35) also gives high dimensional analogue of Hamilton's entropy monotonicity for Kähler-Ricci flow. It still remains a open problem if Hamilton's entropy formula has high dimensional version. 
Localizing LYH inequalities and monotonicity formulae is important to the study of the singularities. The localization of LYH inequality is not an easy matter in general. Li-Yau's original work provides such a localized estimate. There also exists recent fundamental work of Hamilton $[\mathbf{H 7}]$ in this direction. Here we present the localization of the monotonicity formulae via the heat ball/heat sphere. This consideration can be traced back to the works of $[\mathbf{F u}, \mathbf{W a}]$. It is much related to mean value theorems for harmonic functions and solutions to the heat equation. Here we just include a general formulation for evolving metrics [EKNT].

In $[\mathbf{E K N T}]$, a very general scheme on localizing the monotonicity formulae is developed. It is for any family of metrics evolved by the equation $\frac{\partial}{\partial t} g_{i j}=-2 \kappa_{i j}$. The localization is through the so-called 'heat ball'. More precisely for a smooth positive space-time function $v$, which often is the fundamental solution to the backward conjugate heat equation or the pseudo backward heat kernel' $\hat{H}\left(x_{0}, y, \tau\right)=\frac{e^{-\frac{r^{2}\left(x_{0}, y\right)}{4 \tau}}}{(4 \pi \tau)^{\frac{n}{2}}}\left(\right.$ or $\frac{e^{-\ell(y, \tau)}}{(4 \pi \tau)^{\frac{n}{2}}}$ in the case of Ricci flow), with $\tau=t_{0}-t$, one defines the 'heat ball' by $E_{r}=\left\{(y, t) \mid v \geq r^{-n}\right.$; $\left.t<t_{0}\right\}$. For all interesting cases we can check that $E_{r}$ is compact for small $r$ (cf. [EKNT]). Let $\psi_{r}=\log v+n \log r$. For any 'Li-Yau-Hamilton' quantity $\mathcal{Q}$ we define the local quantity:

$$
P(r):=\int_{E_{r}}\left(\left|\nabla \psi_{r}\right|^{2}+\psi_{r}\left(\operatorname{tr} r_{g} \kappa\right)\right) \mathcal{Q} d \mu_{t} d t .
$$

The finiteness of the integral can be verified by a local gradient estimate. The general form of the theorem, which is proved in Theorem 1 of [EKNT], reads as the following.

THEOREM 3.15. Let $I(r)=\frac{P(r)}{r^{n}}$. Then

$$
\begin{aligned}
I\left(r_{2}\right)-I\left(r_{1}\right)= & -\int_{r_{1}}^{r_{2}} \frac{n}{r^{n+1}} \int_{E_{r}}\left[\left(\left(\frac{\partial}{\partial t}+\Delta-t r_{g} \kappa\right) v\right) \frac{\mathcal{Q}}{v}\right. \\
& \left.+\psi_{r}\left(\frac{\partial}{\partial t}-\Delta\right) \mathcal{Q}\right] d \mu_{t} d t d r .
\end{aligned}
$$

It gives the monotonicity of $I(r)$ in the cases that $\mathcal{Q} \geq 0$, which is ensured by the LYH estimates in the case we shall consider, and both $\left(\frac{\partial}{\partial t}+\Delta-\operatorname{tr} r_{g} \kappa\right) v$ and $\left(\frac{\partial}{\partial t}-\Delta\right) \mathcal{Q}$ are nonnegative. The nonnegativity of $\left(\frac{\partial}{\partial t}+\Delta-\operatorname{tr} r_{g} \kappa\right) v$ comes for free if we chose $v$ to be the 'pseudo backward heat kernel'. The nonnegativity of $\left(\frac{\partial}{\partial t}-\Delta\right) \mathcal{Q}$ follows from the key computation, which we call the pre-LYH equation, in the proof of the corresponding LYH estimate.

There exist certain localizations on the entropy formula (3.31). These localizations are achieved by suitable cut-off functions and are easier than the above consideration via the heat balls. Please see $[\mathbf{N 4}]$ for details. 
3.4. Hypersurface flows. For Gauss curvature flow, define.

$$
\mathcal{E}(t) \doteqdot \int_{M} K \log K d \mu
$$

Its monotonicity was first established by Ben Chow, using the proof of his LYH inequality for Gauss curvature flow. It turns out that it holds for slightly more general flows with a certain integrability condition. (This nice observation is due to Andrews [An2].)

A. The general formulation. Recall the notations $\Phi, \Psi, P$, etc, from the previous section. For the simplicity we assume that the flow speed $\Phi>0$.

THEOREM 3.16. Assume that the speed function $\Psi=\Psi(A)$ is $\alpha$-concave and satisfies that

$$
\bar{\nabla}_{i}\left(\Psi^{-2} \dot{\Psi}^{i j}\right)=0
$$

Then

$$
\frac{d}{d t} \int_{\mathbb{S}^{n}} \frac{\partial}{\partial t} \log \Phi d \sigma \geq \frac{\alpha-1}{\alpha} \int_{\mathbb{S}^{n}}\left(\frac{\partial}{\partial t} \log \Phi\right)^{2} d \sigma .
$$

Proof. The proof follows from the computation in previous sections on the LYH estimate for hypersurface flows. Recall that $P=\Psi_{t}$ satisfies that

$$
\frac{\partial}{\partial t} P=\mathcal{L} P+\dot{\Psi}(\mathrm{id}) P+\ddot{\Psi}(Q, Q)
$$

where $Q=\frac{\partial}{\partial t} A=\bar{g}^{*} \operatorname{Hess}_{\bar{\nabla}} \Psi+\Psi$ id. Hence

$$
\begin{aligned}
\frac{d}{d t} \int_{\mathbb{S} n} \frac{\partial}{\partial t} \log \Phi d \sigma & =\int_{\mathbb{S} n} \frac{\frac{\partial}{\partial t} P}{\Psi}-\frac{P \frac{\partial}{\partial t} \Psi}{\Psi^{2}} d \sigma \\
& =\int_{\mathbb{S}^{n}} \frac{(\dot{\Psi})^{i j} \bar{\nabla}_{i} \bar{\nabla}_{j} P+\dot{\Psi}(\mathrm{id}) P}{\Psi}+\frac{\ddot{\Psi}(Q, Q)}{\Psi}-\left(\frac{P}{\Psi}\right)^{2} d \sigma \\
& \geq \int_{\mathbb{S}^{n}} \frac{(\dot{\Psi})^{i j} \bar{\nabla}_{i} \bar{\nabla}_{j} P+\dot{\Psi}(\mathrm{id}) P}{\Psi}+\frac{\alpha-1}{\alpha}\left(\frac{P}{\Psi}\right)^{2}-\left(\frac{P}{\Psi}\right)^{2} d \sigma
\end{aligned}
$$

noting that $\dot{\Psi}(Q)=P$. Now we compute the first term

$$
\begin{aligned}
\int_{\mathbb{S}^{n}} \frac{(\dot{\Psi})^{i j} \bar{\nabla}_{i} \bar{\nabla}_{j} P}{\Psi} d \sigma & =\int_{\mathbb{S}^{n}} \Psi\left(\Psi^{-2}(\dot{\Psi})^{i j} \bar{\nabla}_{i} \bar{\nabla}_{j} P\right) d \sigma \\
& =-\int_{\mathbb{S}^{n}} \bar{\nabla}_{i} \Psi\left(\Psi^{-2}(\dot{\Psi})^{i j} \bar{\nabla}_{j} P\right) d \sigma \\
& =\int_{\mathbb{S}^{n}}\left(\bar{\nabla}_{j} \bar{\nabla}_{i} \Psi\right) \Psi^{-2}(\dot{\Psi})^{i j} P d \sigma
\end{aligned}
$$


Using that $P=(\dot{\Psi})^{i j}\left(\bar{\nabla}_{i} \bar{\nabla}_{j} \Psi+\Psi \bar{g}_{i j}\right)$ we have that

$$
\int_{\mathbb{S}^{n}} \frac{(\dot{\Psi})^{i j} \bar{\nabla}_{i} \bar{\nabla}_{j} P+\dot{\Psi}(\mathrm{id}) P}{\Psi} d \sigma=\int_{\mathbb{S}^{n}} \frac{P^{2}}{\Psi^{2}} d \sigma .
$$

Now we check that the condition (3.39) holds for both the Gauss curvature flow and harmonic mean curvature flow.

Gauss curvature flow: Using the normal coordinate centered at a point, we have that $A=\left(A_{i j}\right)$ with $A_{i j}=\bar{\nabla}_{i} \bar{\nabla}_{j} s+s \bar{g}_{i j}$. Direct calculation shows that

Hence it suffices to show

$$
\Psi^{-2}(\dot{\Psi})^{i j}=\operatorname{det}(A)\left(A^{-1}\right)_{i j}=\frac{\partial \operatorname{det}(A)}{\partial A_{i j}} .
$$

$$
\bar{\nabla}_{i}\left(\frac{\partial \operatorname{det}(A)}{\partial A_{i j}}\right)=0 .
$$

This follows from two claims below.

1) For any symmetric tensor $A$ satisfying the Codazzi equation $\bar{\nabla}_{k} A_{i j}=\bar{\nabla}_{i} A_{j k}$, we have

$$
\bar{\nabla}_{i}\left(\frac{\partial S_{k}(A)}{\partial A_{i j}}\right)=0
$$

where $S_{k}$ is the $k$-th symmetric function.

2) The $A=\left(\bar{\nabla}_{i} \bar{\nabla}_{j} s+s \bar{g}_{i j}\right)$ satisfies the Codazzi equation.

The first claim can be checked directly. For the second one, we have the following computation.

$$
\begin{aligned}
\bar{\nabla}_{k} & \left(\bar{\nabla}_{i} \bar{\nabla}_{j} s+s \bar{g}_{i j}\right)-\bar{\nabla}_{i}\left(\bar{\nabla}_{k} \bar{\nabla}_{j} s+s \bar{g}_{k j}\right) \\
& =\bar{\nabla}_{k} \bar{\nabla}_{i} \bar{\nabla}_{j} s-\bar{\nabla}_{i} \bar{\nabla}_{k} \bar{\nabla}_{j} s+\bar{\nabla}_{k} s \bar{g}_{i j}-\bar{\nabla}_{i} s \bar{g}_{k j} \\
& =-\bar{R}_{i k j p} \bar{\nabla}_{p} s+\bar{\nabla}_{k} s \bar{g}_{i j}-\bar{\nabla}_{i} s \bar{g}_{k j} \\
& =0
\end{aligned}
$$

where we have used the expression for the curvature $\bar{R}_{i k j p}=\bar{g}_{i j} \bar{g}_{k p}-\bar{g}_{i p} \bar{g}_{k j}$.

Harmonic mean curvature flow: Direct calculation shows that $\Psi^{-2}$ $(\dot{\Psi})^{i j}=\bar{g}_{i j}$. Hence $\bar{\nabla}_{i}\left(\Psi^{-2}(\dot{\Psi})^{i j}\right)$ is trivially true.

B. The entropy formulae. Now we look further into the Gauss curvature flow case. Noting that $\mathcal{E}=\int_{\mathbb{S} n} \log K d \sigma$, a consequence of the theorem via the Hölder inequality is that

$$
\frac{d^{2} \mathcal{E}(t)}{d t^{2}} \geq \frac{n+1}{n A\left(\mathbb{S}^{n}\right)}\left(\frac{d \mathcal{E}(t)}{d t}\right)^{2}
$$


Now consider the normalized flow. Let

$$
\tilde{X}=\psi^{-1}(t) X
$$

where $\psi=((n+1)(T-t))^{\frac{1}{n+1}}$. Hence the new equation is

$$
\frac{\partial}{\partial \tau} \tilde{X}=-\tilde{K} \nu+\tilde{X}
$$

where $\tau=\frac{1}{n+1} \log \left(1-\frac{t}{T}\right)$. It can be checked that the flow preserves the enclosed volume if $V\left(M_{0}\right)=\omega_{n+1}$, the volume of the unit ball. Now the corresponding equation on the support function $\tilde{s}$ is that

$$
\frac{\partial}{\partial \tau} \tilde{s}=\tilde{s}-\tilde{K}=\tilde{s}-\frac{1}{\operatorname{det}\left(\bar{g}^{*} \operatorname{Hess}_{\bar{\nabla}} \tilde{s}+\tilde{s} \text { id }\right)} .
$$

Let $\tilde{A}=\bar{g}^{*}\left(\operatorname{Hess}_{\bar{\nabla}} \tilde{s}+\bar{g} \tilde{s}\right)$. Now

$$
\frac{\partial}{\partial \tau} \tilde{A}=\tilde{A}+\bar{g}^{*}\left(\operatorname{Hess}_{\bar{\nabla}} \tilde{\Psi}+\operatorname{id} \tilde{\Psi}\right) .
$$

Similarly $\tilde{P} \doteqdot \frac{\partial}{\partial \tau} \tilde{\Psi}=\dot{\tilde{\Psi}}\left(\frac{\partial}{\partial \tau} \tilde{A}\right)$ satisfies

$$
\begin{aligned}
\frac{\partial}{\partial \tau} \tilde{P} & =\mathcal{L} \tilde{P}+\dot{\tilde{\Psi}}(\mathrm{id}) \tilde{P}+\mathcal{L} \tilde{\Psi}+\dot{\tilde{\Psi}}(\mathrm{id}) \tilde{\Psi}+\dot{\tilde{\Psi}}(\tilde{A})+\ddot{\tilde{\Psi}}(\tilde{Q}, \tilde{Q}) \\
& =\mathcal{L} \tilde{P}+\dot{\tilde{\Psi}}(\mathrm{id}) \tilde{P}+\dot{\tilde{\Psi}}\left(\frac{\partial}{\partial \tau} \tilde{A}\right)+\ddot{\tilde{\Psi}}(\tilde{Q}, \tilde{Q}) \\
& =\mathcal{L} \tilde{P}+\dot{\tilde{\Psi}}(\mathrm{id}) \tilde{P}+P+\tilde{\tilde{\Psi}}(\tilde{Q}, \tilde{Q})
\end{aligned}
$$

where $\tilde{Q}=\frac{\partial}{\partial \tau} \tilde{A}$. Let $\tilde{\mathcal{E}}(\tau)=\int_{\mathbb{S}^{n}} \log \tilde{K} d \sigma$. Repeating the calculation in the last subsection we have that

$$
\frac{d^{2} \tilde{\mathcal{E}}(\tau)}{d \tau^{2}} \geq \frac{n+1}{n A\left(\mathbb{S}^{n}\right)}\left(\frac{d \tilde{\mathcal{E}}(\tau)}{d \tau}\right)^{2}+\frac{d \tilde{\mathcal{E}}(\tau)}{d \tau}
$$

which then implies the following result of Chow. Note that

$$
\tilde{\mathcal{E}}(\tau)=\int_{\mathbb{S}^{n}} \log \left(((n+1)(T-t))^{\frac{n}{n+1}} K(x, t)\right) d \sigma .
$$

Theorem 3.17 .

$$
\frac{d \tilde{\mathcal{E}}(\tau)}{d \tau} \leq 0
$$

Proof. This is derived out of the long time existence result of Tso $[\mathbf{T}]$. Otherwise, assume that $\mathcal{F} \doteqdot \frac{d \tilde{\mathcal{E}}}{d \tau}>0$ (abbreviated as $\tilde{\mathcal{E}}^{\prime}$ ) at some $\tau_{0}$. By (3.43) we have that $\mathcal{F}^{\prime} \geq \alpha \mathcal{F}^{2}$ for all $\tau \geq \tau_{0}$. This implies that $\mathcal{F}$ must blow up at some finite time. A contradiction! 
This derivation of the monotonicity formula via the long time existence and ODE consideration is originated in [H2].

It is also worthwhile to look into the special case that the entropy is constant. Tracing the proof we conclude that $\tilde{K}_{\tau}=0$ and

$$
\tilde{A}=\lambda \bar{g}^{*}\left(\operatorname{Hess}_{\bar{\nabla}} \tilde{\Psi}+\bar{g} \tilde{\Psi}\right) .
$$

Using the PDE satisfied by $\tilde{\Psi}$, it is easy to conclude that the above $\lambda=-1$, which implies $\frac{\partial}{\partial \tau} \tilde{A}=0$. In particular we have that $\operatorname{Hess}_{\bar{\nabla}}(\tilde{K}-\tilde{s})+(\tilde{K}-\tilde{s}) \bar{g}=$ 0 . This concludes that $\tilde{K}-\tilde{s}$ is the restriction of a linear function. Keeping in mind that the choices of different origin to define the support function cause the support function to differ by a linear function, we essentially have that $\tilde{s}-\tilde{K}=0$. Namely the equality holds only on a shrinking soliton (steady solution to the normalized flow).

The following dual version of the entropy formula was motivated by the thermodynamic consideration as before. Define the following entropy-like quantities:

$$
\begin{aligned}
\overline{\mathcal{E}}(t) & =-\int_{\mathbb{S}^{n}} \log \left(t^{\frac{n}{n+1}} K\right) d \sigma \\
\mathcal{W}(t) & =\frac{d}{d t}(t \overline{\mathcal{E}}(t))=t \frac{d}{d t} \overline{\mathcal{E}}(t)+\overline{\mathcal{E}}(t) \\
& =-t \frac{d}{d t} \mathcal{E}(t)-\frac{n A\left(\mathbb{S}^{n}\right)}{n+1}+\overline{\mathcal{E}}(t) .
\end{aligned}
$$

By the LYH inequality we have that

$$
\frac{d}{d t} \overline{\mathcal{E}}(t) \leq 0
$$

Letting $\mathcal{K}=\frac{\partial}{\partial t} \log K$, the above computation also gives

$$
\begin{aligned}
\frac{d}{d t} \mathcal{W}(t)= & -t \frac{d}{d t}\left(\frac{d \mathcal{E}(t)}{d t}\right)-2 \frac{d \mathcal{E}(t)}{d t}-\frac{n A\left(\mathbb{S}^{n}\right)}{(n+1) t} \\
& \leq-\int_{\mathbb{S}^{n}}\left(\frac{n+1}{n} t \mathcal{K}^{2}+2 \mathcal{K}+\frac{n}{(n+1) t}\right) d \sigma \\
& \leq-\frac{n t}{n+1} \int_{\mathbb{S}^{n}}\left(\frac{n+1}{n} \mathcal{K}+\frac{1}{t}\right)^{2} d \sigma .
\end{aligned}
$$

The following corollary summarizes the above observations.

Corollary 3.18. The entropies $\overline{\mathcal{E}}(t)$ and $\mathcal{W}(t)$ are monotone nonincreasing.

Similar entropy formulae for $\overline{\mathcal{E}}(t)$ and $\mathcal{W}(t)$, which can be similarly defined as

$$
\overline{\mathcal{E}}(t)=-\int_{\mathbb{S}^{n}} \log \left(\Phi t^{\frac{\alpha}{\alpha-1}}\right) d \sigma \quad \text { and } \quad \mathcal{W}(t)=\frac{d}{d t}(t \overline{\mathcal{E}}(t))
$$


can be shown verbatim provided that the assumption of Theorem 3.16 holds, particularly including the harmonic mean curvature flow.

By tracing the equality in either (3.45) or (3.46) we have that

$$
\frac{K_{t}}{K}+\frac{n}{t(n+1)}=0
$$

and

$$
-Q=\bar{g}^{*}\left(\bar{\nabla}_{i} \bar{\nabla}_{j} K+K \bar{g}_{i j}\right)=\lambda A
$$

for some $\lambda$ which may depend on space-time point. Using the equation for $K$ one deduces that $\lambda=-\frac{1}{(n+1) t}$. Hence as before we conclude that the equality holds only if $K+\frac{1}{(n+1) t} s=0$. Namely the solution is an expanding soliton.

\section{The other direction}

The previous section shows how LYH inequality implies the monotonicity formulae. This process sometimes can be reversed. Here we show that some geometric considerations which lead to the monotonicity of some geometric quantities also suggest the LYH inequalities (2.5) (for the heat equation) and (2.14) (for Ricci flow) respectively. According to Perelman, it is this consideration that leads the discovery of his entropy formula and (2.14).

4.1. Linear heat equation. Recall that the LYH inequality (2.42) implies (2.8), which is equivalent to the following result of Cheeger and Yau, which asserts that on a complete Riemannian manifold with nonnegative Ricci curvature, the heat kernel $H(x, \tau ; o, 0)$ (the fundamental solution of the operator $\left(\frac{\partial}{\partial \tau}-\Delta\right)$ ) has the lower estimate

$$
H(x, \tau ; o, 0) \geq \frac{1}{(4 \pi \tau)^{\frac{n}{2}}} \exp \left(-\frac{r^{2}(o, x)}{4 \tau}\right)
$$

where $r(o, x)$ is the distant function on the manifold. This fact can be derived out of the maximum principle and the differential inequality

$$
\left(\frac{\partial}{\partial \tau}-\Delta\right)\left(\frac{1}{(4 \pi \tau)^{\frac{n}{2}}} \exp \left(-\frac{r^{2}(o, x)}{4 \tau}\right)\right) \leq 0 .
$$

Integrating on the manifold $M$, this differential inequality also implies the monotonicity (monotone non-increasing) of the integral

$$
\tilde{V}(o, \tau):=\int_{M} \frac{1}{(4 \pi \tau)^{\frac{n}{2}}} \exp \left(-\frac{r^{2}(o, y)}{4 \tau}\right)
$$


Fix a point $o \in M$. Let $\gamma(\tau)(0 \leq \tau \leq \bar{\tau})$ be a curve parameterized by the time variable $\tau$ with $\gamma(0)=o$. Here we image that we have a time function $\tau$, with which some parabolic equation is associated. Define the $\mathcal{L}$-length by

$$
\mathcal{L}(\gamma)(\bar{\tau})=\int_{0}^{\bar{\tau}} \sqrt{\tau}\left|\gamma^{\prime}(\tau)\right|^{2} d \tau
$$

We can define the $\mathcal{L}$-geodesic to be the curve which is the critical point of $\mathcal{L}(\gamma)$. The simple computation shows that the first variation of $\mathcal{L}$ is given by

$$
\delta \mathcal{L}(\gamma)=2 \sqrt{\bar{\tau}}\langle Y, X\rangle(\bar{\tau})-2 \int_{0}^{\bar{\tau}} \sqrt{\tau}\left(\left\langle\nabla_{X} X+\frac{1}{2 \tau} X, Y\right\rangle\right) d \tau,
$$

where $Y$ is the variational vector field, from which one can write down the $\mathcal{L}$-geodesic equation. It is an easy matter to see that $\gamma$ is a $\mathcal{L}$-geodesic if and only if $\gamma(\sigma)$ with $\sigma=2 \sqrt{\tau}$ is a geodesic. In other words, a $\mathcal{L}$-geodesic is a geodesic after certain re-parametrization. Here we insist all curves are parameterized by the 'time'-variable $\tau$. One can check that for any $v \in$ $T_{o} M$ there exists a $\mathcal{L}$-geodesic $\gamma(\tau)$ such $\left.\frac{d}{d \sigma}(\gamma(\sigma))\right|_{\sigma=0}=v$. Notice that the variable $\sigma$ scales in the same manner as the distance function on $M$. So it is more convenient to work with $\sigma$.

Following $[\mathbf{P}]$ we also introduce the $\ell$-'distance' function.

$$
\left.\ell_{o}(y, \bar{\tau})=\frac{1}{2 \sqrt{\bar{\tau}}} L_{o}(y, \bar{\tau}), \quad \text { where } L_{o}(y, \bar{\tau})=\inf _{\gamma} \mathcal{L}(\gamma)\right) .
$$

Here our $\ell$ is defined for a fixed background metric. We also omit the subscript $o$ in the context where the meaning is clear. The similar computations as in $[\mathbf{P}]$, together with the second variation formula from the Riemannian geometry, show that

$$
\begin{gathered}
|\nabla \ell|^{2}=\frac{1}{\bar{\tau}} \ell \\
\ell_{\tau}=-\frac{1}{\bar{\tau}} \ell
\end{gathered}
$$

and

$$
\Delta \ell \leq \frac{n}{2 \bar{\tau}}-\frac{1}{\bar{\tau}^{\frac{3}{2}}} \int_{0}^{\bar{\tau}} \tau^{\frac{3}{2}} \operatorname{Ric}(X, X) d \tau
$$

where $X=\gamma^{\prime}(\tau)$ with $\gamma(\tau), 0 \leq \tau \leq \bar{\tau}$ being the minimizing $\mathcal{L}$-geodesic joining $o$ to $x$. Putting (4.5)-(4.7) together, one obtains a new proof of the result of Cheeger-Yau, which asserts that if $M$ has nonnegative Ricci curvature, then

$$
-\ell_{\tau}+\Delta \ell-|\nabla \ell|^{2}-\frac{n}{2 \tau} \leq 0
$$


which is equivalent to

$$
\left(\frac{\partial}{\partial \tau}-\Delta\right)\left(\frac{e^{-\ell(x, \tau)}}{(4 \pi \tau)^{\frac{n}{2}}}\right) \leq 0 .
$$

Namely $\frac{e^{-\ell(x, \tau)}}{(4 \pi \tau)^{\frac{n}{2}}}$ is a sub-solution of the heat equation. In particular,

$$
\frac{d}{d \tau} \int_{M} \frac{e^{-\ell(x, \tau)}}{(4 \pi \tau)^{\frac{n}{2}}} d \mu \leq 0 .
$$

Using the above geometric consideration, one can think of the above result of Cheeger-Yau as a parabolic volume comparison with respect to the positive measure $\frac{e^{-\ell(x, \tau)}}{(4 \pi \tau)^{\frac{n}{2}}} d \mu$. Recall that the well-known Bishop volume comparison states that if $M$ has nonnegative Ricci curvature

$$
\frac{d}{d r}\left(\frac{1}{r^{n-1}} \int_{S_{x_{0}}(r)} d A\right) \leq 0
$$

where $S_{o}(r)$ denotes the boundary of the geodesic ball centered at $o$ with radius $r, d A$ is the induced area measure. The by-now standard relative volume comparison can be formulated in the similar way as above. Let $A$ be a measurable subset of $S^{n-1} \subset T_{o} M$ one can define $C_{A}(r)$ to be the collection of vectors $r v$ with $v \in A$ such that the geodesic $\exp _{o}(s v)$ is minimizing for $s \leq r$, where $\exp _{o}(\cdot)$ is the exponential map. Then the relative volume comparison theorem asserts that if $M$ has nonnegative Ricci curvature

$$
\frac{d}{d r}\left(\frac{1}{r^{n-1}} \int_{\exp \left(C_{A}(r)\right)} d A\right) \leq 0 .
$$

The following is a parabolic version of such a relative volume comparison theorem parallel to Perelman's work on Ricci flow geometry.

Proposition 4.1. Assume that $M$ has nonnegative Ricci curvature. Then

$$
\frac{d}{d \tau} \int_{\mathcal{L} \exp _{C_{A}(\tau)}(\tau)} \frac{e^{-\ell(x, \tau)}}{(4 \pi \tau)^{\frac{n}{2}}} d \mu \leq 0 .
$$

Better comparison between Theorem 4.1 and the classical relative volume comparison can be seen by identifying the space-time $\widetilde{M}=M \times[0, T]$ with the manifold $M$, the time-slice $M \times\{a\}$ with the geodesic sphere $S_{o}(a)$. Notice that in $[\mathbf{P}]$, one does need such a localized version (for Ricci flow) to prove the important no local collapsing result on the finite time solution to Ricci flow. 
The other combinations of (4.5)-(4.7) include the Hamilton-Jacobi equations:

$$
|\nabla \ell|^{2}+2 \ell_{\tau}+\frac{\ell}{\tau}=0
$$

$$
|\nabla \ell|^{2}+\ell_{\tau}=0
$$

and the inequalities

$$
\begin{gathered}
\Delta \bar{L}+\bar{L}_{\tau} \leq 2 n, \quad \text { for } \bar{L}=4 \tau \ell, \\
\tau\left(2 \Delta \ell-|\nabla \ell|^{2}\right)+\ell-n \leq 0 .
\end{gathered}
$$

Notice that assuming (4.10), (4.8) and (4.13) imply each other. One way of thinking is that $\ell$ as a solution to a Hamilton-Jacobi equation (4.10), one has (4.13) and (4.8). On the other hand Theorem 2.2 asserts that for the fundamental solution $u=\frac{e^{-\ell}}{(4 \pi \tau)^{\frac{n}{2}}}$ to the heat equation, one has (4.13). Namely, if we insist the equality in (4.8) (hence we look at solutions to the heat equation in stead of the Hamilton-Jacobi equation (4.10)) we still have (4.13). The proof of this observation leads to the entropy formula (3.15). One should also compare (4.10) with (2.6), (4.11) with (2.3), (4.12) with $(2.9)$.

4.2. Ricci flow. We find it most striking that the above geometric consideration has a close analogue for the space-time of the solution to the backward Ricci flow $\frac{\partial}{\partial \tau} g=\operatorname{Ric}(g)$. This is one of the most important contributions of $[\mathbf{P}]$.

Recall (from(2.47), a consequence of the LYH (2.42)) the definition of reduced distance

$$
\ell_{x_{0}, g(\tau)}(x, \bar{\tau}):=\inf _{\gamma} \frac{1}{2 \sqrt{\tau}} \int_{0}^{\bar{\tau}} \sqrt{\tau}\left(\left|\gamma^{\prime}(\tau)\right|^{2}+R\right) d \tau
$$

for all $\gamma(\tau)$ with $\gamma(0)=x_{0}, \gamma(\bar{\tau})=x$ (in the right context, we often omit the subscript $\left.x_{0}, g(\tau)\right)$. The quantity

$$
\tilde{V}_{g(\tau)}\left(x_{0}, \tau\right):=\int_{M} \frac{1}{(4 \pi \tau)^{\frac{n}{2}}} \exp (-\ell(x, \tau)) d \mu(\tau)
$$

is called the reduced volume. Perelman proved that $\tilde{V}_{g(\tau)}\left(x_{0}, \tau\right)$ is monotone non-increasing in $\tau$. It was proved in $[\mathbf{P}]$ by the first and second variation 
consideration that

$$
\begin{aligned}
\ell_{\tau} & =R-\frac{\ell}{\tau}+\frac{1}{2 \tau^{\frac{3}{2}}} K, \\
|\nabla \ell|^{2} & =-R+\frac{\ell}{\tau}-\frac{1}{\tau^{\frac{3}{2}}} K, \\
\Delta \ell & \leq-R+\frac{n}{2 \tau}-\frac{1}{2 \tau^{\frac{3}{2}}} K,
\end{aligned}
$$

where $K=K(\gamma, \bar{\tau})=\int_{0}^{\bar{\tau}} \tau^{\frac{3}{2}} H(X) d \tau$, with $\gamma$ being the minimizing $\mathcal{L}$ geodesic, $X=\gamma^{\prime}(\tau)$ and

$$
H(X)=-R_{\tau}-\frac{R}{\tau}-2\langle X, \nabla R\rangle+2 \operatorname{Ric}(X, X) .
$$

Grouping the above in different ways we have that

$$
\begin{array}{r}
2 \ell_{\tau}+|\nabla \ell|^{2}-R+\frac{\ell}{\tau}=0, \\
-\ell_{\tau}+\Delta \ell-|\nabla \ell|^{2}+R-\frac{n}{2 \tau} \leq 0, \\
\tau\left(2 \Delta \ell-|\nabla \ell|^{2}+R\right)+\ell-n \leq 0,
\end{array}
$$

as well as

$$
\Delta \bar{L}+\bar{L}_{\tau} \leq 2 n
$$

with $\bar{L}=4 \tau \ell$. The monotonicity of the reduced volume follows from (4.20) easily.

Considering the first and second variation of energy functional as (4.14) and applying it to prove the monotonicity of a quantity similar as (4.15) were originated in the seminar work of Li and Yau $[\mathbf{L Y}]$ on the Schrödinger operator. As in the linear heat equation case, there they have to assume that the Ricci curvature is nonnegative together with other conditions on the potential function of the Schrödinger equation. The most remarkable thing is that for the backward Ricci flow space time, the whole thing fits together without assuming anything on the curvature.

Note that assuming (4.19), the inequalities (4.20) and (4.21) imply each other and each of them can be viewed as a preserved inequality for the solution to the Hamilton-Jacobi equation (4.19) (The stressing of the HamiltonJacobi equation is attributed to $[\mathbf{H 8}]$ for the Ricci flow case and the much earlier work $[\mathbf{L Y}]$ for the linear Schrödinger equation). According to a conversation with Perelman, by insisting that (4.20) holds equality (namely solving a conjugate heat equation), Perelman discovered the LYH inequality (4.21), namely (2.42), for the fundamental solution. The proof of this leads to the entropy formula (3.31). Later on Hamilton [H8] observed that if one insists on equation (4.19), then for the solution to this Hamilton-Jacobi type 
equation, (4.21) will be preserved. Based on this he also [H8] proposed a more general notion of reduced distance and claimed some interesting monotonicity and relations between entropy and the reduced volume. We found that this duality is quite interesting and deserves further understanding.

Naturally one would ask if the similar consideration can lead to an entropy-like formula for the hypersurface flow.

4.3. Dual version and the matrix inequality. The same line of thinking as the above can motivate the matrix LYH in Theorem 2.10. This time one considers the reduced geometry of the Ricci flow (instead of the backward Ricci flow). For the sake of the exposition we shall focus on KählerRicci flow.

Let $g(t)$ be a complete solution to Kähler-Ricci flow on $M^{m} \times[0, T]$ (where $m=\operatorname{dim}_{\mathbb{C}}(M)$ and $n=2 m$ ). Fix $x_{0}$ and let $\gamma$ be a path $(x(\eta), \eta)$ joining $\left(x_{0}, 0\right)$ to $(x, \bar{t})$. Following $[\mathbf{P}]$ (see also $\left.[\mathbf{L Y}, \mathbf{F I N}]\right)$ we define

$$
\mathcal{L}_{+}(\gamma)=\int_{0}^{\bar{t}} \sqrt{t}\left(R+4\left|\gamma^{\prime}(t)\right|^{2}\right) d t
$$

Let $X=\gamma^{\prime}(t)=\frac{d z^{\alpha}(t)}{d t} \frac{\partial}{\partial z^{\alpha}}$ and let $Y$ be a variational vector field along $\gamma$. Here $\left|\gamma^{\prime}(t)\right|^{2}=g_{\alpha \bar{\beta}} \frac{d z^{\alpha}(t)}{d t} \frac{d z^{\bar{\beta}}(t)}{d t}$. Using $\mathcal{L}_{+}$as energy we can define the $\mathcal{L}_{+^{-}}$ geodesics and we denote $L_{+}(y, t)$ to be the length of a shortest geodesics jointing $\left(x_{0}, 0\right)$ to $(y, t)$. We also define

$$
\ell_{+}\left(x, t ; x_{0}, 0\right):=\frac{1}{2 \sqrt{t}} L_{+}(x, t)
$$

Following the first and second variation calculation of $[\mathbf{P}]$ (see also $[\mathbf{F I N}]$ ) we have that

$$
\begin{aligned}
\left|\nabla \ell_{+}\right|^{2} & =-R+\frac{\ell_{+}}{t}+\frac{K}{t^{3 / 2}}, \\
\frac{\partial \ell_{+}}{\partial t} & =R-\frac{K}{2 t^{3 / 2}}-\frac{\ell_{+}}{t}, \\
\Delta \ell_{+} & \leq R+\frac{n}{2 t}-\frac{K}{2 t^{3 / 2}} .
\end{aligned}
$$

Here

$$
K:=\int_{0}^{t} \eta^{3 / 2} H(X) d \eta,
$$

where $H(X):=\partial R / \partial t+2\langle\nabla R, X\rangle+2\langle X, \nabla R\rangle+4 \operatorname{Ric}(X, X)+R / t$, is exactly the traced LYH differential Harnack expression in $[\mathbf{C o}]$ applying to the $(1,0)$ vector field $2 X$. 
Grouping (4.24)-(4.26) suitably we have the following

$$
\begin{aligned}
2 \frac{\partial \ell_{+}}{\partial t}+\left|\nabla \ell_{+}\right|^{2}-R+\frac{\ell_{+}}{t} & =0, \\
\frac{\partial \ell_{+}}{\partial t}+\Delta \ell_{+}+\left|\nabla \ell_{+}\right|^{2}-R-\frac{n}{2 t} & \leq 0, \\
\left(\frac{\partial}{\partial t}-\Delta\right)\left(\bar{L}_{+}+2 n t\right) & \geq 0 \\
t\left(2 \Delta \ell_{+}+\left|\nabla \ell_{+}\right|^{2}-R\right)-\ell_{+}-n & \leq 0 .
\end{aligned}
$$

In particular (4.30) suggests an entropy formula, which is dual to Perelman's (3.31) since it holds equality on expanding solitons.

THEOREM 4.2. Let $(M, g(t))$ be a solution to Ricci flow. Let $u$ be a solution to the conjugate heat equation with $\int_{M} u=1$. Write $u=\frac{e^{-f_{+}}}{\left(4 \pi\left(t-t_{0}\right)\right)^{\frac{n}{2}}}$ for $t>t_{0}$. Let

$$
\mathcal{W}_{+}=\int_{M}\left[\left(t-t_{0}\right)\left(\left|\nabla f_{+}\right|^{2}+R\right)-f_{+}+n\right] u d \mu_{t} .
$$

Then

$$
\frac{\partial \mathcal{W}_{+}}{\partial t}=2\left(t-t_{0}\right) \int_{M}\left|R_{i j}+\nabla_{i} \nabla_{j} f_{+}+\frac{g_{i j}}{2\left(t-t_{0}\right)}\right|^{2} d \mu
$$

Note that the entropy expression is suggested by applying (4.30) to $\hat{u}=\frac{e^{\ell}(x, t)}{\left(4 \pi\left(t-t_{0}\right)\right)^{\frac{n}{2}}}$, which is a super-solution of the conjugate heat equation by $(4.28)$.

If we assume that $(M, g)$ has nonnegative bisectional curvature, by regrouping and observing that $K \geq 0$ we have the following result.

ThEOREM 4.3. Let $\left(M^{m}, g(t)\right)$ be a complete solution to Kähler-Ricci (Ricci) flow with bounded nonnegative bisectional curvature (curvature operator $)$. Let $H\left(y, t ; x_{0}, 0\right)$ be the fundamental solution to forward conjugate heat equation centered at $\left(x_{0}, 0\right)$. Then

$$
\tilde{u}(x, t):=\frac{1}{(\pi t)^{m}} \exp \left(-\ell_{+}\left(x, t ; x_{0}, 0\right)\right)
$$

satisfies

$$
\left(\frac{\partial}{\partial t}-\Delta-R\right) \tilde{u}(x, t) \leq 0
$$

In particular,

$$
\tilde{u}\left(x, t ; x_{0}, 0\right) \leq H\left(x, t ; x_{0}, 0\right)
$$


and

$$
\tilde{\theta}_{+}^{\left(x_{0}, 0\right)}(t):=\int_{M} \tilde{u}(x, t) d \mu_{t}(x)
$$

is monotone decreasing. Moreover, the equality in (4.32), or (4.33) implies that $M$ is a gradient expanding soliton.

ProOF. First (4.24)-(4.26) implies that

$$
\begin{array}{r}
\left(\frac{\partial}{\partial t}-\Delta-R\right)\left(\frac{1}{(\pi t)^{m}} \exp \left(-\ell_{+}(x, t)\right)\right) \\
=-\frac{K}{t^{\frac{3}{2}}}\left(\frac{1}{(\pi t)^{m}} \exp \left(-\ell_{+}(x, t)\right)\right) \leq 0 .
\end{array}
$$

Here we have used the fact that $K \geq 0$ under the assumption that $M$ has bounded non-negative bisectional curvature. Also if the equality holds it implies that $K \equiv 0$. This further implies that $M$ is an expanding soliton from the computation in $[\mathbf{F I N}]$. In order to prove (4.33) one just needs to apply the maximum principle and notice that $\lim _{t \rightarrow 0} \frac{1}{(\pi t)^{m}} \exp \left(-\ell_{+}(x, t)\right)=$ $\delta_{x_{0}}(x)$. The equality case follows from the analysis on the equality case in [FIN].

Moreover we also have the following observation which motivated Theorem 2.10.

Proposition 4.4. Assume that $(M, g(t))$ be a Kähler-Ricci flow with bounded nonnegative bisectional curvature on $M \times[0, T)$. Let $\tilde{u}(x, t)$ be as in Theorem 4.3. Then

$$
\log (\tilde{u})_{\alpha \bar{\beta}}+R_{\alpha \bar{\beta}}+\frac{1}{t} g_{\alpha \bar{\beta}} \geq 0
$$

The equality holds if and only if $(M, g(t))$ is an expanding Kähler-Ricci solition.

\section{Comments}

Due to the limited time allowed and the lack of expertise we could not address in this exposition many important aspects related to LYH inequalities. Among them, the most notable is the space-time consideration of Chow and $\mathrm{Chu}[\mathbf{C C 1}, \mathbf{C C 2}, \mathbf{C C 3}]$, and the very recent work of Hamilton on the local approximation of the trace LYH inequality for the Ricci flow [H7]. The main theme of the space-time consideration is to interpret the LYH expression as the curvature operator of certain degenerate metric on the space time $\mathcal{M}=M \times[0, T]$. This geometric consideration sometimes suggests new LYH estimates $[\mathbf{C K}, \mathbf{C h e}]$. A similar space-time construction for the backward Ricci flow was done by Perelman $[\mathbf{P}]$. This construction for the backward Ricci flow is related to the reduced distance and monotonicity of the reduced volume. 
Concerning the monotonicity, we completely missed the monotonicity of many important energy functionals, such as the Donaldson functional and the Mabuchi energy, constructed in the study of Hermitian metrics on holomorphic vector bundles or Kähler metrics/potentials. They seem not related to LYH type estimates in general.

\section{References}

[An1] B. Andrews, Contraction of convex hypersurfaces in Euclidean space, Calc. Var. 2(1994), 151-171.

[An2] B. Andrews, Harnack inequalities for evolving hypersurfaces, Math. Z. 217(1994), 179-197.

[B] S. Bando, On the classification of three-dimensional compact Kaehler manifolds of nonnegative bisectional curvature, J. Differential Geom. 19(1984), no. 2, 283-297.

[Co] H.-D. Cao, On Harnack inequalities for the Kähler-Ricci flow, Invent. Math. 109(1992), 247-263, MR1172691, Zbl 0779.53043.

[CN] H.-D. Cao and L. Ni, Matrix Li-Yau-Hamilton estimates for heat equation on Kähler manifolds, Math. Ann. 331(2005), 795-807, MR2148797, Zbl pre02156245.

[CY] J. Cheeger and S.-T. Yau, A lower bound for the heat kernel, Comm. Pure Appl. Math. 34(1981), no. 4, 465-480, MR0615626, Zbl 0481.35003.

[ChY1] S. Y. Cheng and S. T. Yau, Differential equations on Riemannian manifolds and their geometric applications, Comm. Pure Appl. Math. 28(1975), no. 3, 333-354.

[ChY2] S. Y. Cheng and S. T. Yau, Maximal space-like hypersurfaces in the LorentzMinkowski spaces, Ann. of Math. (2) 104(1976), no. 3, 407-419.

[Che] H.-B. Cheng, A New Li-Yau-Hamilton Estimate for the Ricci Flow, Comm. Anal. Geom. 14(2006), 551-564.

[Ch1] B. Chow, On Harnack's inequality and entropy for the Gaussian curvature flow, Comm. Pure Appl. Math. 44(1991), no. 4, 469-483.

[Ch2] B. Chow, The Yamabe flow on locally conformally flat manifolds with positive Ricci curvature, Comm. Pure Appl. Math. 45(1992), no. 8, 1003-1014.

[Ch3] B. Chow, Interpolating between Li-Yau's and Hamilton's Harnack inequalities on a surface, J. Partial Differential Equations 11(1998), no. 2, 137-140, MR1626999, Zbl 0943.58017.

[CC1] B. Chow and S.-C. Chu, A geometric interpretation of Hamilton's Harnack inequality for the Ricci flow, Math. Res. Lett. 2(1995), no. 6, 701-718, MR1362964, Zbl 0856.53030 .

[CC2] B. Chow and S.-C. Chu, A geometric approach to the linear trace Harnack inequality for the Ricci flow, Math. Res. Lett. 3(1996), no. 4, 549-568, MR1406020, Zbl 0868.58082 .

[CC3] B. Chow and S.-C. Chu, Space-time formulation of Harnack inequalities for curvature flow of hypersurfaces, J. Geom. Anal. 11(2001), 219-231.

$[\mathrm{CH}]$ B. Chow and R. Hamilton, Constrained and linear Harnack inqualities for parabolic equations, Invent. Math. 129(1997), 213-238, MR1465325.

[CK] B. Chow and D. Knopf, New Li-Yau-Hamilton inequalities for the Ricci flow via the space-time approach, J. Differential Geom. 60(2002), no. 1, 1-54, MR1924591, Zbl 1048.53026 .

[E1] K. Ecker, A local monotonicity formula for mean curvature flow, Ann. of Math. (2) 154(2001), no. 2, 503-525, MR1865979, Zbl 1007.53050.

[E2] K. Ecker, Regularity Theory for Mean Curvature Flow, Progress in Nonlinear Differential Equations and their Applications 57. Birkhuser Boston, Inc., Boston, MA, 2004, MR2024995, Zbl 1058.53054. 
[EKNT] K. Ecker, D. Knopf, L. Ni and P. Topping, Local monotonicity and mean value formulas for evolving Riemannian manifolds, to appear in Crelle's Journal.

[FIN] M. Feldman, T. Ilmanen and $\mathrm{L}$. Ni, Entropy and reduced distance for Ricci expanders, J. Geom. Anal. 15(2005), 49-62, MR2132265, Zbl 1071.53040.

[Fu] W. B. Fulks, A mean value theorem for heat equation, Proc Amer. Math Soc. 17(1966), 6-11.

[H1] R. Hamilton, Four-manifolds with positive curvature operator, J. Differenital. Geom. 24(1986), 153-179.

[H2] R. Hamilton, The Ricci flow on surfaces, Mathematics and general relativity (Santa Cruz, CA, 1986), 237-262, Contemp. Math., 71, Amer. Math. Soc., Providence, RI, 1988.

[H3] R. Hamilton, The Harnack estimate for the Ricci flow, J. Differential Geom. 37(1993), no. 1, 225-243, MR1316556, Zbl 0804.53023.

[H4] R. Hamilton, Harnack estimate for the mean curvature flow, J. Differential Geom. 41(1995), no. 1, 215-226.

[H5] R. Hamilton, A matrix Harnack estimate for the heat equation, Comm. Anal. Geom. 1(1993), no. 1, 113-126, MR1230276, Zbl 0799.53048.

[H6] R. Hamilton, Monotonicity formulas for parabolic flows on manifolds, Comm. Anal. Geom. 1(1993), no. 1, 127-137, MR1230277, Zbl 0779.58037.

[H7] R. Hamilton, A local approximate Harnack estimate for the Ricci flow, preprint, 2006.

[H8] R. Hamilton, Lectures on Perelman's reduced distance and differential Harnack inequality.

[Hu] G. Huisken, Asymptotic behavior for singularities of the mean curvature flow, J. Differential Geom. 31(1990), no. 1, 285-299.

[HS] G. Huisken and C. Sinestrari, Convexity estimates for mean curvature flow and singularities of mean convex surfaces, Acta Math. 183(1999), 45-70.

[KM] K. Kodaira and J. Morrow, Complex Manifolds. Holt, Rinehart and Winston, Inc. 1971.

[LY] P. Li and S.-T. Yau, On the parabolic kernel of the Schrödinger operator, Acta Math. 156(1986), no. 3-4, 153-201, MR0834612, Zbl 0611.58045.

[Mo] J. Moser, A Harnack inequality for parabolic differential equations, Comm. Pure Appl. Math. 17(1964), 101-134, MR0159139, Zbl 0149.06902.

[N1] L. Ni, Monotonicity and Kähler-Ricci flow, Contemp. Math. 367(2005), 149-165, MR2115758.

[N2] L. Ni, The entropy formula for linear heat equation, J. Geom. Anal. 14(2004), 87-100, MR2030576, Zbl 1062.58028; addenda, J. Geom. Anal. 14(2004), 369-374, MR2051693.

[N3] L. Ni, A monotonicity formula on complete Kähler manifold with nonnegative bisectional curvature, J. Amer. Math. Soc. 17(2004), 909-946, MR2083471, Zbl pre02106879.

[N4] L. Ni, A matrix Li-Yau-Hamilton inequality for Kähler-Ricci flow, J. Differential Geom. 75(2007), 303-358.

[N5] L. Ni, A note on Perelman's LYH type inequality, Comm. Anal. Geom. 14(2006), $883-905$.

[NT] L. Ni and L.-F. Tam, Plurisubharmonic functions and the Kähler-Ricci flow, Amer. J. Math. 125(2003), 623-654, MR1981036, Zbl 1027.53076.

[P] G. Perelman, The entropy formula for the Ricci flow and its geometric applications, arXiv: math.DG/ 0211159.

[SY] R. Schoen and S. T. Yau, Lectures on differential geometry. Lecture notes prepared by Wei Yue Ding, Kung Ching Chang [Gong Qing Zhang], Jia Qing Zhong and Yi Chao $\mathrm{Xu}$. Translated from the Chinese by Ding and S. Y. Cheng. Preface translated from the Chinese by Kaising Tso. Conference Proceedings and Lecture Notes in Geometry and Topology, I. International Press, Cambridge, MA, 1994. v+235 pp. 
[T] K.-S. Chou (Kaiseng Tso), Deforming a hypersurface by its Gauss-Kronecker curvature, Comm. Pure Math. Appl. 38(1985), 867-882.

[Wa] N. A. Watson, A theory of temperatures in several variables, Proc. London Math. Soc. 26(1973), 385-417.

[Y] S. T. Yau, Harmonic functions on complete Riemannian manifolds, Comm. Pure Appl. Math. 28(1975), 201-228.

Department of Mathematics, University of California at San Diego, LA Jolla, CA 92093

E-mail address: lni@math.ucsd.edu 
